\title{
Coexistence of Weak and Strong Wave Turbulence in Incompressible Hall Magnetohydrodynamics
}

\author{
Romain Meyrand, ${ }^{1,2, *}$ Khurom H. Kiyani, ${ }^{2,3}$ Özgur D. Gürcan, ${ }^{2}$ and Sébastien Galtier ${ }^{2,4}$ \\ ${ }^{1}$ Space Sciences Laboratory, University of California, Berkeley, California 94720, USA \\ ${ }^{2}$ LPP, CNRS, École Polytechnique, Univ. UPMC, Univ. Paris-Sud, Observatoire de Paris, Université \\ Paris-Saclay, Sorbonne Université, PSL Research University, 91128 Palaiseau, France \\ ${ }^{3}$ Centre for Fusion, Space and Astrophysics, University of Warwick, Coventry CV4 7AL, United Kingdom \\ ${ }^{4}$ Department of Physics, University of Paris-Sud, Orsay 91400, France
}

(Received 18 April 2018; revised manuscript received 26 June 2018; published 12 September 2018; corrected 6 December 2018)

\begin{abstract}
We report a numerical investigation of three-dimensional, incompressible, Hall magnetohydrodynamic turbulence with a relatively strong mean magnetic field. Using helicity decomposition and crossbicoherence analysis, we observe that the resonant three-wave coupling is substantial among ion-cyclotron and whistler waves. A detailed study of the degree of nonlinearity of these two populations shows that the ion-cyclotron component experiences a transition from weak to strong wave turbulence going from large to small scales, while the whistler fluctuations display a weak wave turbulence character for all scales. This nontrivial coexistence of the two regimes with two populations of waves gives rise to anomalous anisotropy and scaling properties. The weak and strong wave turbulence components can be distinguished rather efficiently using spatiotemporal Fourier transforms. The analysis shows that while resonant triad interactions survive the highly nonlinear bath of ion-cyclotron fluctuations at large scales for which the degree of nonlinearity is low for both populations of waves, whistler waves tend to be killed by the nonlinear cross-coupling at smaller scales where the ion-cyclotron component is in the strong wave turbulent regime. Such a situation may have far-reaching implications for the physics of magnetized turbulence in many astrophysical and space plasmas and probably beyond, where different waves coexist and compete to transfer energy nonlinearly, across scales.
\end{abstract}

DOI: 10.1103/PhysRevX.8.031066

Subject Areas: Plasma Physics

\section{INTRODUCTION}

Turbulence is an ubiquitous dynamical phenomenon involving many spatial and temporal scales. It manifests itself in many problems involving the evolution of a fluid system. Except a few special cases, this multiscale dynamics happens in the presence of external factors, such as a background magnetic field, or rotation, giving rise to waves, instabilities, kinetic or other dissipation mechanisms, whose existence affects the underlying nonlinear mechanisms of energy transfer. This general case can be approached with different idealizations. One such idealization is to neglect linear terms completely, including waves, and consider the Kolmogorov picture of turbulence where the nonlinear cascade takes place in a pure inertial range. Another idealization is weak turbulence theory (WTT), where the

*omain.meyrand@lpp.polytechnique.fr

Published by the American Physical Society under the terms of the Creative Commons Attribution 4.0 International license. Further distribution of this work must maintain attribution to the author(s) and the published article's title, journal citation, and DOI. energy is transferred across scales only through resonant interactions among waves. Note that any real physical system that can support waves is almost always in a mixed state between a pure inertial cascade and a pure wave turbulence cascade. Here, we consider Hall-MHD as a clean, simple example of a generic mixed problem, where different components of such a cascade can be identified.

A sea of weakly nonlinear dispersive waves can exchange energy among spatial scales, resulting in a highly turbulent state. Because of the weak nonlinear coupling, the energy transfer takes place mainly via resonant interactions among a set of waves. The resulting behavior for a statistically stationary state, far from thermodynamic equilibrium, bears resemblance to the cascade picture in threedimensional (3D) hydrodynamic turbulence: Energy injected at large scales cascades toward smaller scales where dissipation irreversibly transforms it into heat. A fundamental difference from strong hydrodynamic turbulence is that an out-of-equilibrium system made of weakly interacting waves is free of the closure difficulties and thus appears to be a solvable problem for which rigorous analytical predictions can be made in the framework of WTT [1]. WTT was developed during the 1960s with the 
pioneering works on gravity waves traveling at the surface of the ocean [2]. Soon after, the theory was applied to plasma physics [3] and, since then, to an increasing number of physical problems, ranging from quantum [4] to cosmological systems [5].

While the idealization described by WTT deals only with weakly nonlinear random waves, in most real situations, weakly nonlinear waves and strongly nonlinear coherent structures coexist. In 3D magnetohydrodynamic (MHD) turbulence, because 2D vortices play a catalytic role for the triadic interactions of Alfvén waves, nonlinear coherent structures have a strong impact on the weak wave turbulent dynamics [6]. A similar situation, where coherent structures have a large impact on weak wave dynamics, arises in nonequilibrium Bose-Einstein condensation [7] or in optical turbulence [8] as well.

Another possible complication is that the dominant waves change their character as a function of scale. This is the case, for example, at the surface of water, where gravity waves transform at a small scale into capillary waves [9] under the influence of surface tension. Similarly, in rotating turbulence, the effect of the Coriolis force, which decreases as a function of scale, may lead to the conversion of inertial waves into highly nonlinear fluctuations at the so-called Rhines scale. Because of these "real life" effects, experiments often show deviations from the existing predictions, and as a result, weak wave turbulence is rarely observed in its pure form [10]. However, in these examples, the coherent nonlinear structures and/or the different weak wave fields do not "live" in the same part of the spectral range, which facilitates the analytical and experimental disentangling of the two components.

In plasma physics, the situation seems to be different because an entire zoo of various waves can effectively coexist at any given scale. The question then is, how do these different waves coexist and compete to transfer energy across scales? In this paper, we address this question using a simple archetypal example of plasma turbulence modeled by the incompressible Hall MHD equations. In the framework of Hall MHD, the electrons are assumed to be inertialess, and the electric field is determined by the equation of motion of the electron fluid. As a result, the magnetic field is frozen-in to the electrons (modulo Ohmic losses) and not to the bulk fluid. At sub-ion scales, this decoupling leads to the emergence of two circularly polarized waves with opposite polarity, the so-called whistler and ion-cyclotron waves. The kinetic equations for three-wave interaction processes describing the nonlinear dynamics of weak wave Hall MHD turbulence were derived in the general case, i.e., including the nonlinear interactions between different types of waves. The exact power-law solutions were also derived analytically [11] but only in the simplified case where the interactions between the ion-cyclotron and whistler waves are negligible. One important aim of this article is to study in detail to what extent this assumption is justified.
In Sec. II, we describe the incompressible Hall MHD approximation and present the associated equations. Section III constitutes the core of the article. We present results from high-resolution 3D direct numerical simulations. In particular, we show that the ion-cyclotron waves experience a transition from weak to strong wave turbulence, going from large to small scales, while the whistler fluctuations display a weak wave turbulence character for all scales. In Sec. IV, we first show that resonant triadic interactions survive the highly nonlinear bath of ion-cyclotron fluctuations; we then show that the three-wave coupling is substantial among ion-cyclotron and whistler waves, using higher-order polyspectra techniques. In Sec. V, we focus on the properties of the space-time Fourier spectrum, which allows us to show that the resonant triadic interactions are at work at large scales for which both whistler and ioncyclotron fluctuations are weakly nonlinear, while whistlers are killed by local cross-coupling with strongly nonlinear ion-cyclotron fluctuations at smaller scales. We give our conclusion in Sec. VI, where this and other findings are discussed along with their implications.

\section{INCOMPRESSIBLE HALL MHD}

Hall MHD is a theoretical paradigm that captures both the MHD behavior at long wavelengths and some of the kinetic effects that become important at small scales due to the decoupling between the electron and ion flows. This can be done by keeping the Hall current term in the ideal Ohm's law (in SI units):

$$
\mathbf{E}+\mathbf{u} \times \mathbf{B}-\frac{\mathbf{j} \times \mathbf{B}}{n e}-\frac{\nabla p_{e}}{n e}=0,
$$

where $\mathbf{E}$ is the electric field, $\mathbf{u}$ the bulk velocity, $\mathbf{j}$ the electric current, $\mathbf{B}$ the magnetic field, $n$ the electron density, $e$ the magnitude of the electron charge, and $p_{e}$ the electron pressure. The Hall term becomes dominant at length scales smaller than the ion inertial length $d_{i}\left(d_{i} \equiv c / \omega_{p i}\right.$, with $c$ being the speed of light and $\omega_{p i}$ being the ion plasma frequency) and timescales of the order of, or shorter than, the ion-cyclotron period $\omega_{c i}^{-1}$.

The linear dispersion relation of the Hall MHD can be recovered exactly from the full kinetic dispersion relation in the limit of $T_{i} \ll T_{e}, v_{\text {thi }} \ll \omega /\left|k_{\|}\right| \ll v_{\text {the }}$, and $\omega \ll \omega_{c i}$ [12,13], with $T_{i, e}$ and $v_{\text {thi,the }}$ respectively the ion or electron temperature and thermal speed. In other words, Hall MHD is a valid approximation under the condition that the parameter regime considered is such that it is rigorously justified to completely ignore collisionless damping, cyclotron resonance, and finite Larmor radius effects. Examples of plasmas where it may hold are cold and dense regions of protoplanetary discs [14] and crusts of neutron stars [15], as well as some plasma research devices like the Madison Plasma Dynamo experiment [16], the Wisconsin Plasma Astrophysics Laboratory [17], 
the Magnetic Reconnection Experiment [18,19], or the levitated dipole experiment [20,21].

In the context of solar wind turbulence, the cold ion limit has been flagged as an important limitation of Hall MHD because spurious undamped wave modes appear when the ion temperature is finite [22] and because the spectral break and the associated change in the nature of the turbulent cascade in Hall MHD turbulence may appear at the wrong scale $\left(d_{i}\right.$ instead of $\left.\rho_{i}\right)$ [23]. Concerning the first argument, it is important to recall two points. First, robust Kolmogorov-like power-law spectra of compressible fluctuations in the inertial range of solar wind turbulence are frequently observed $[24,25]$, even though Landau damping of such fluctuations should be noticeable at these scales [23]. The same phenomenon has been observed at sub-ion scales [26,27] and in some kinetic simulations [28]. These observations show that collisionless damping rates derived from linear kinetic theory are not applicable in a turbulent plasma. Second, because of the anisotropy, the (linearly damped) compressive perturbations have a tendency to be passively mixed by the undamped Alfvénic turbulence [29]. Consequently, even if the linear kinetic theory is applicable to plasma turbulence, the existence of certain (undamped) wave modes in Hall MHD that are linearly damped in a weakly collisional plasma would not necessarily affect the nonlinear dynamics. Concerning the second argument, in situ measurements of magnetic fluctuations in the solar wind show that the spectral break at ion kinetic scales occurs either at $d_{i}$ or $\rho_{i}$ depending on the value of $\beta_{i}$ [30], showing that different physical processes are at work and that it is difficult to lock up this complex phenomenon in a complete and exclusive theory. More generally, if one considers that the salient feature of plasma turbulence is a flux of invariants through scales rather than thermodynamic potentials like temperature, it might appear that a fluid model like Hall MHD can provide useful insights in the study of plasma turbulence without bringing in the full complexity of the kinetic theory.

The (inviscid and ideal) incompressible 3D Hall MHD equations can be obtained from incompressible MHD if one introduces the generalized Ohm's law (1) into MaxwellFaraday's equation and assumes that the electron pressure $p_{e}$ is a scalar (this can be justified in the collisional limit or in the isothermal electron fluid approximation [23]). This gives

$$
\begin{gathered}
\nabla \cdot \mathbf{u}=0, \quad \nabla \cdot \mathbf{b}=0, \\
\frac{\partial \mathbf{u}}{\partial t}+\mathbf{u} \cdot \nabla \mathbf{u}=-\nabla P_{*}+\mathbf{b}_{0} \cdot \nabla \mathbf{b}+\mathbf{b} \cdot \nabla \mathbf{b}, \\
\frac{\partial \mathbf{b}}{\partial t}+\mathbf{u} \cdot \nabla \mathbf{b}=\left(\mathbf{b}_{0} \cdot \nabla\right)\left(\mathbf{u}-d_{i} \nabla \times \mathbf{b}\right)+\mathbf{b} \cdot \nabla \mathbf{u} \\
-d_{i} \nabla \times[(\nabla \times \mathbf{b}) \times \mathbf{b}],
\end{gathered}
$$

where $P_{*}$ is the total pressure, $\mathbf{b}$ is the magnetic field normalized to a velocity $\left(\mathbf{b}=\mathbf{B} / \sqrt{\mu_{0} n m_{i}}\right.$, with $m_{i}$ the ion mass), and $\mathbf{b}_{0}$ is a uniform normalized magnetic field. The assumption of incompressibility allows us to drop the sonic wave, which is thought to be less relevant because of its damping by kinetic effects [31]; therefore, we can accurately describe the two remaining dispersive branches of compressible Hall MHD at finite $\beta$ values [32]. Note that Eq. (4) in the limit $k d_{i} \gg 1$ and $k_{\perp} \gg k_{\|}$is mathematically similar to the electron reduced MHD (ERMHD) equations to within a constant coefficient, which is probably not essential for qualitative models of turbulence [23]. Thus, a detailed study of the mechanisms of cascade in the incompressible Hall MHD is useful for understanding kinetic Alfvén wave cascade as well.

\section{SPECTRAL PROPERTIES: ANISOTROPY, SCALING, TRANSFER, AND FLUX}

\section{A. Simulation setup}

We solve the incompressible Hall MHD equations in a periodic, rectangular domain with aspect ratio $L_{\perp}^{2} \times L_{\|}$ using the TURBO code [33], in which we have implemented the Hall term. A series of benchmarks, including those against exact nonlinear solutions [34] of the Hall MHD equations, are presented in Ref. [35]. We set $L_{\perp}=L_{\|}=2 \pi$, $d_{i}=0.5$, and $b_{0}=25$. A 3D pseudospectral algorithm is used to perform the spatial discretization on a grid with a resolution of $N_{\perp}^{2} \times N_{\|}$mesh points (see Table I). The time step is computed automatically by a Courant-FriedrichsLewy $\mathrm{CFL}=0.3$ criterion, and the time advancement is based on a modified Williamson, four-step, third-order, lowstorage Runge-Kutta method [36]. To save on computational costs, we have reduced the field-parallel numerical resolution with $N_{\perp}>N_{\|}$. This is appropriate since the energy cascade proceeds much faster in the field-perpendicular direction, resulting in an anisotropy in $\mathbf{k}$ space such that the energy at large $k_{\|}$is reduced.

The initial state consists of isotropic magnetic and velocity field fluctuations with random phases such that the total cross helicity $H^{c}=\langle\mathbf{u} \cdot \mathbf{b}\rangle$, the total magnetic helicity $H^{m}=\langle\mathbf{a} \cdot \mathbf{b}\rangle$, and the kinetic helicity $H^{k}=$ $\langle\mathbf{u} \cdot \nabla \times \mathbf{u}\rangle$ are zero $(\langle\cdots\rangle$ denotes a volume average). The initial kinetic and magnetic energies are equal to $1 / 2$

TABLE I. Summary of the simulations parameters.

\begin{tabular}{lcrcc}
\hline \hline Run & $N_{\perp}$ & \multicolumn{1}{c}{$N_{\|}$} & $\nu_{3}$ & $\eta_{3}$ \\
\hline I Hall MHD & 768 & 512 & $5 \times 10^{-13}$ & $2 \times 10^{-11}$ \\
II Hall MHD & 768 & 256 & $1.7 \times 10^{-3}$ & $2 \times 10^{-11}$ \\
III Hall MHD & 128 & 64 & $1.7 \times 10^{-9}$ & $6.4 \times 10^{-9}$ \\
IV Hall MHD & 256 & 128 & $7.6 \times 10^{-11}$ & $4.5 \times 10^{-10}$ \\
V EMHD & 256 & 128 & $\varnothing$ & $4.5 \times 10^{-10}$ \\
\hline \hline
\end{tabular}


and localized at the largest scales of the system (wave numbers $|\mathbf{k}| d_{i} \in[1,2]$ are initially excited).

For the purpose of this study, we have developed a helical forcing. The turbulence is driven at the largest scales with a forcing that is local in Fourier space. It acts on all the modes within the shell $s_{f}$ defined by $|\mathbf{k}| d_{i} \in[1.25,1.75]$. In practice, the kinetic $\hat{\mathbf{f}}_{u}$ and magnetic $\hat{\mathbf{f}}_{b}$ forces have the form

$$
\begin{aligned}
\hat{\mathbf{f}}_{u}(\mathbf{k}) & =\sum_{\Lambda} \alpha_{\Lambda}(\mathbf{k}) \mathcal{U}_{\Lambda}(\mathbf{k}) \mathbf{h}_{k}^{\Lambda}, \\
\hat{\mathbf{f}}_{b}(\mathbf{k}) & =\sum_{\Lambda} \beta_{\Lambda}(\mathbf{k}) \mathcal{B}_{\Lambda}(\mathbf{k}) \mathbf{h}_{k}^{\Lambda},
\end{aligned}
$$

where $\mathcal{U}_{\Lambda}$ and $\mathcal{B}_{\Lambda}$ correspond to the Fourier transform of the original vectors $\mathbf{u}(\mathbf{x})$ and $\mathbf{b}(\mathbf{x})$ projected on a complex helicity basis (see Appendix A). The parameters $\alpha_{\Lambda}(\mathbf{k})$ and $\beta_{\Lambda}(\mathbf{k})$ are given by

$$
\begin{aligned}
& \alpha_{\Lambda}(\mathbf{k})=\frac{\varepsilon_{\Lambda}^{u}}{E_{\Lambda}^{u}(\mathbf{k}) N_{f}^{2}}, \\
& \beta_{\Lambda}(\mathbf{k})=\frac{\varepsilon_{\Lambda}^{b}}{E_{\Lambda}^{b}(\mathbf{k}) N_{f}^{2}},
\end{aligned}
$$

where $2 E_{\Lambda}^{u}(\mathbf{k})=\left|\mathcal{U}_{\Lambda}(\mathbf{k})\right|^{2}, 2 E_{\Lambda}^{b}(\mathbf{k})=\left|\mathcal{B}_{\Lambda}(\mathbf{k})\right|^{2}$, and $N_{f}$ is the number of forced modes. This choice ensures that each of the $N_{f}$ forced modes is submitted to a forcing mechanism that injects kinetic and magnetic energy at the constant rates $\sum_{\Lambda} \varepsilon_{\Lambda}^{u}$ and $\sum_{\Lambda} \varepsilon_{\Lambda}^{b}$. We fix $\varepsilon_{+}^{u}=\varepsilon_{-}^{u}=\varepsilon_{+}^{b}=$ $\varepsilon_{-}^{b}=0.025$, which forces the kinetic helicity and magnetic helicity injection rates to be zero. Remarkably, this choice imposes a cross-helicity level close to zero. Note that in the momentum equation (3), the kinetic forcing $\mathbf{f}^{u}$, whatever its precise form, can always be considered as divergence-free since the pressure will enforce the incompressibility of the velocity field by eliminating any $\nabla \cdot \mathbf{f}^{u}$ contribution of the force. On the other hand, $\mathbf{f}^{b}$ must always be divergence-free as a consistency condition for the magnetic field. This latter condition is automatically satisfied by the helical nature of the forcing. Importantly, since the forces are proportional to the fields, the characteristic time of the forces will tend to be equal to that of the intrinsic characteristic time of the large-scale eddies (corresponding to modes within the shell $s_{f}$ ). With such a forcing, we therefore do not introduce any artificial and potentially dynamically disturbing characteristic times. Furthermore, since the $\alpha$ and $\beta$ parameters are real, the forcing method presented here does not influence the phases of the fields, which ensures that no change is made in the type of turbulent structures present.

The system is evolved until a stationary state is reached for both the velocity and the magnetic fields, which is confirmed by observing the time evolution of the total energy as well as the dissipation rate of total energy of the fluctuations (not shown). Note that, in order to achieve a stationary state, it is necessary to remove the amount of ideal invariants that may be injected into the system by the forcing mechanism. In order to achieve this, we use kinetic hyperdissipation $\nu_{3} \Delta^{3}$ and magnetic hyperdiffusivity $\eta_{3} \Delta^{3}$. When the stationary state is reached, the kinetic and magnetic energy fluxes relax to a level constrained by the kinetic hyperdissipation and magnetic hyperdiffusivity, respectively. Note that since the non linearities involves respectively a double and single spacial derivative for the magnetic field and the velocity field, it is necessary if one want to have equal dissipative scales for both fields, to choose different value for $\nu_{3}$ and $\eta_{3}$.

As shown in Table I, we conducted a number of runs to investigate various aspects of $3 \mathrm{D}$ incompressible Hall MHD turbulence.

\section{B. Domain of validity of WTT}

WTT deals with asymptotic developments that are based on a timescale separation, with a nonlinear time assumed to be much larger than the wave period. Consequently, a necessary condition for the existence of weak turbulence is that the ratio between linear and nonlinear timescales is small compared to 1 . We therefore evaluate the turbulence regime by considering the different timescales of the problem. In Hall MHD, because two waves with two different dispersion relations exist at sub-ion scales, it is necessary to define two different nonlinear timescales. Because the left-handed ion-cyclotron waves are associated with the velocity field (see discussion in Appendix A 3), we may define the corresponding left-handed nonlinear timescale from the momentum equation (3) as $\tau_{\mathrm{nl}}^{L} \sim 1 /\left(k_{\perp} u_{L}\right)$. $A$ contrario because the right-handed whistler waves are associated with the magnetic field, we may define the righthanded nonlinear timescale from the Maxwell-Faraday's equation (4) as $\tau_{\mathrm{nl}}^{R} \sim 1 /\left(d_{i} k_{\perp}^{2} b_{R}\right)$. Therefore, the asymptotic condition $\tau_{\text {tr }} \gg \tau_{w}$ of WTT implies that the following two relations are fulfilled simultaneously:

$$
\left\{\begin{array}{l}
\chi_{L}^{u}=\frac{\tau_{c i}}{\tau_{\mathrm{nl}}^{L}} \sim \frac{d_{i} k_{\perp}^{2} u_{L}}{k_{\|} b_{0}} \ll 1, \\
\chi_{R}^{b}=\frac{\tau_{w}}{\tau_{\mathrm{nl}}^{R}} \sim \frac{k_{\perp} b_{R}}{k_{\|} b_{0}} \ll 1,
\end{array}\right.
$$

where $\tau_{c i} \sim d_{i} k_{\perp} /\left(k_{\|} b_{0}\right)$ and $\tau_{w} \sim 1 /\left(d_{i} b_{0} k_{\|} k_{\perp}\right)$. It is clear from these two equations that the WTT is not uniformly valid in all of $\mathbf{k}$ space and that its range of applicability can be different for $L$ and $R$ turbulent fluctuations. If we substitute the WTT predictions for the kinetic and magnetic energy spectra in Eq. (9), we can estimate the $k_{\perp}$ dependence of $\chi_{L}^{u}$ and $\chi_{R}^{b}$. It gives

$$
\left\{\begin{array}{l}
\chi_{L}^{u} \propto k_{\perp}^{5 / 4}, \\
\chi_{R}^{b} \propto k_{\perp}^{1 / 4} .
\end{array}\right.
$$


This means that the degree of nonlinearity of left-handed ion-cyclotron fluctuations increases much more rapidly than the degree of nonlinearity of right-handed whistler fluctuations.

The plots of $\chi_{L}^{u}$ and $\chi_{R}^{b}$ corresponding to run I are given in Fig. 1 for different values of $k_{\|}$. For this evaluation, $u_{L}$ and $b_{R}$ are, respectively, defined as $b_{R}=\sqrt{2 k_{\|} k_{\perp} E_{R}^{b}\left(k_{\|}, k_{\perp}\right)}$ and $u_{L}=\sqrt{2 k_{\|} k_{\perp} E_{L}^{u}\left(k_{\|}, k_{\perp}\right)}$. The axisymmetric bidimensional magnetic and kinetic spectra $E^{b, u}\left(k_{\|}, k_{\perp}\right)$ are linked to the magnetic and kinetic energies $\mathcal{E}^{b, u}$ of the system through the relation $\mathcal{E}^{b, u}=\iint E^{b, u}\left(k_{\|}, k_{\perp}\right) d k_{\perp} d k_{\|}$. We clearly see that the $R$ fluctuations belong to the weak turbulence regime for all $k_{\perp}$ and $k_{\|}>0$. For the $L$ fluctuations, the situation is radically different. There exists a critical scale around $k_{\perp} d_{i} \sim 6$ beyond which the weak turbulence cascade drives itself into a state that no longer satisfies the premise on which the theory is based. Note that a similar situation is excepted in Alfvén wave turbulence and has been observed in direct numerical simulations [37]. If it is true that some $L$ modes belong to the weak turbulence regime for all $k_{\perp}$ (those for which $\left.k_{\|}>64\right)$, they actually do not contribute significantly to the

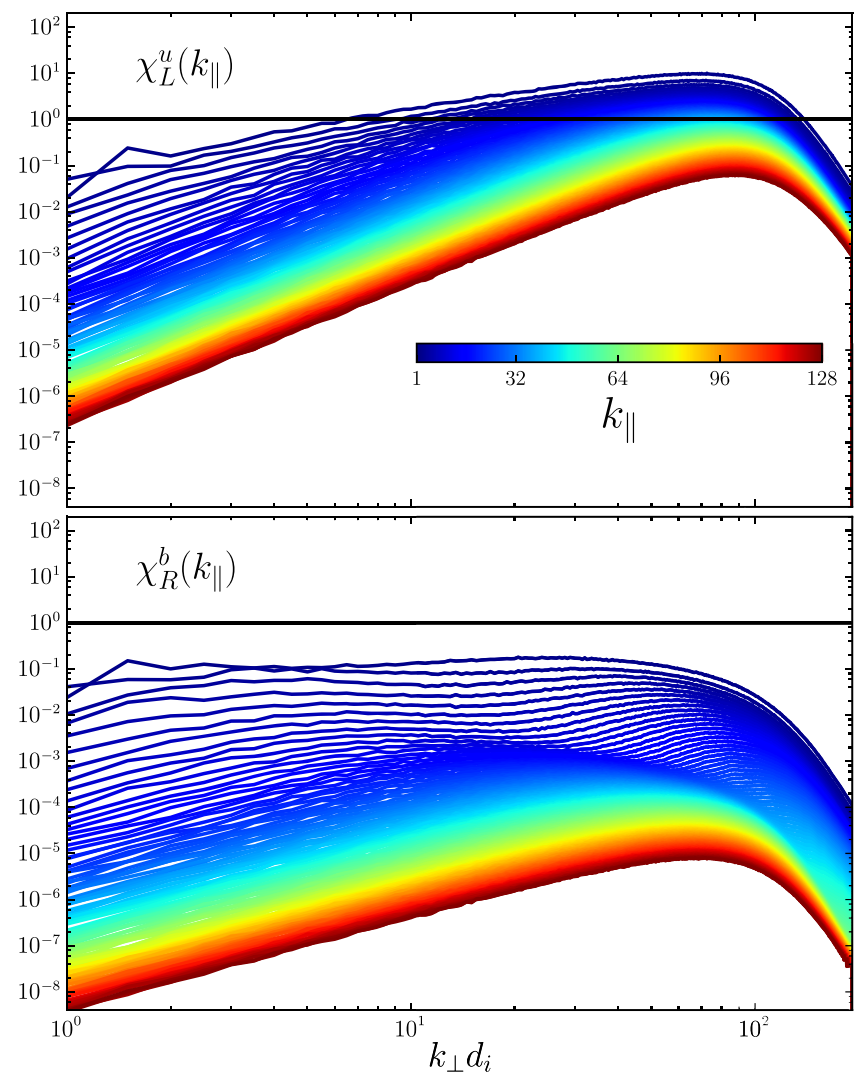

FIG. 1. Transverse wave number dependence of the time ratios $\chi_{L}^{u}$ (top panel) and $\chi_{R}^{b}$ (bottom panel) for different values of $k_{\|}$at $t \sim 90 \omega_{c i}$ (run I). The horizontal line marks the demarcation between weak (below) and strong (above) wave turbulence. dynamics because they are energetically subdominant by several orders of magnitude. The present numerical simulation therefore consists in a nontrivial superposition of mainly highly nonlinear ion-cyclotron modes and weakly nonlinear whistler waves.

In principle, one should be able to obtain a weak turbulent regime for both populations of waves. It is enlightening to evaluate the value of $b_{0}$ necessary to enforce a ion-cyclotron weak wave turbulent regime for every wave number in run I. The transition scale $k_{\perp}^{*}$ is given by $d_{i} k_{\perp}^{2 *} u_{L} / k_{\|} b_{0}=1$. In the weak wave turbulent regime, there is very little energy transfer in the parallel direction, and $k_{\|}$is therefore a parameter corresponding to the $k_{\|}$ forcing scales. Because in weak wave turbulence $u_{L}$ scales as $k_{\perp}^{-3 / 4}, k_{\perp}^{*} \propto\left(k_{\|} b_{0} d_{i}^{-1}\right)^{4 / 5}$. To obtain a pure ion-cyclotron weak wave turbulent regime, the transition scale $k_{\perp}^{*}$ must at least be equal to the largest wave number resolved-so approximately 40 times higher in the case of run I. Therefore, $b_{0}$ must be at least multiplied by a factor $x$ such as $x^{4 / 5}=40$, which gives $b_{0}=25 * 40^{5 / 4}=2500$. By increasing the strength of the mean magnetic field by this factor, the nonlinear frequency broadening becomes smaller than the frequency spacing between adjacent modes. This effect is not taken into account in the WTT because the frequency spacing between adjacent modes is made equal to zero by the so-called infinite size limit at the base of the analytical development. In a finite-size box, discrete effects may have a strong impact on the dynamics when the degree of nonlinearity becomes too small [38]. In this case, the number of quasiresonances can be strongly depleted or absent altogether, and only the terms in the dynamical equations that correspond to exact wave-number and frequency resonances contribute to the nonlinear wave dynamics. In a discrete box, like the one considered in this paper, the $R$ resonant triads become isolated. In this case, the averaged (over the fast linear oscillations) nonlinearity is negligible, and the $R$ weak turbulent cascade over scales is almost completely "frozen." The $L$ field decouples from the magnetic field and displays strong turbulent dynamics. A way to overcome this difficulty would be to elongate the numerical box in the direction of the mean magnetic field, thereby reducing the frequency spacing between adjacent modes. Nevertheless, the cost in terms of computation is prohibitive, and a pure weak turbulence regime for both $L$ and $R$ fluctuations turns out to be inaccessible in practice.

\section{Properties of the energy spectra}

Figure 2 displays the one-dimensional axisymmetric transverse velocity and magnetic spectra (an integration over a cylinder whose axis of symmetry is $\mathbf{b}_{0}$ ) for the $L$ and $R$ fluctuations corresponding to run I at time $t \sim 90 \omega_{c i}$ for which the simulation is statistically stationary. As predicted by the kinematics (see Appendix B), there is a $\xi_{\Lambda}^{-s 2}$ difference between the spectra of the same polarity. 

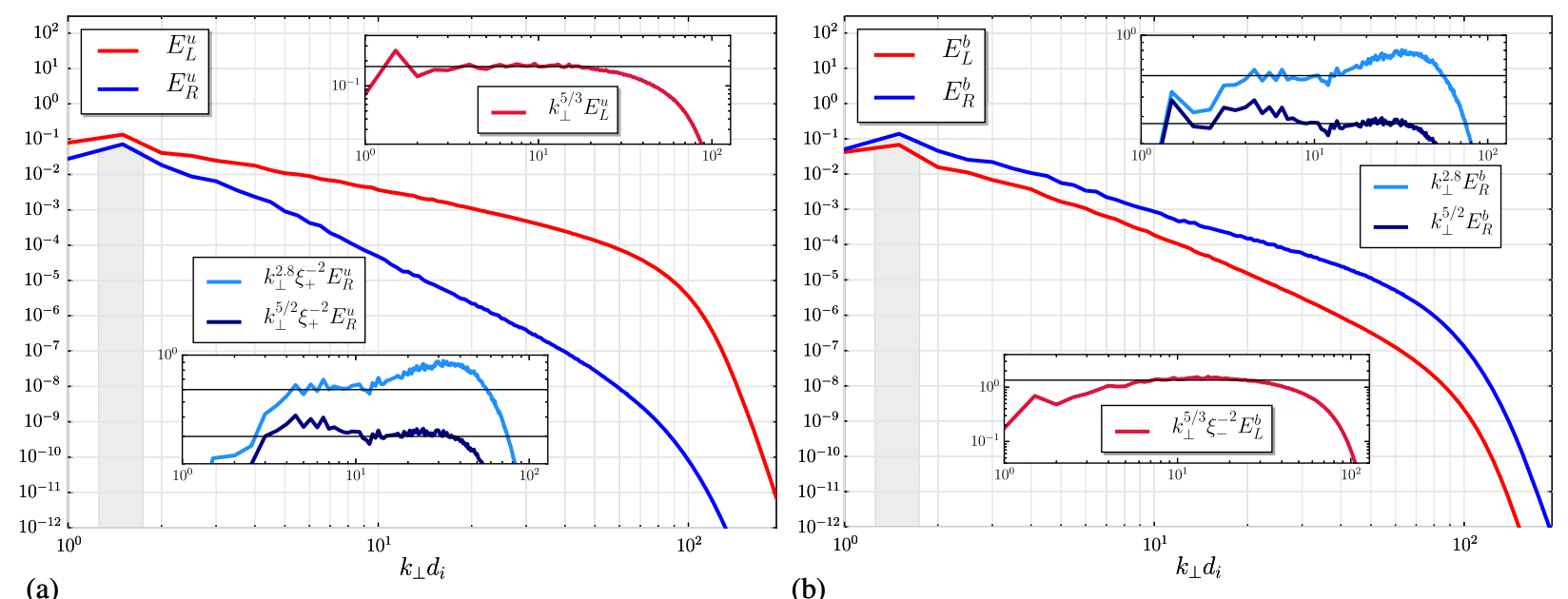

FIG. 2. (a) Transverse velocity spectra of the $L$ fluctuations (red line) and $R$ fluctuations (blue line) (run I). The grey column corresponds to the forcing scales, and the insets show $L$ and $R$ compensated spectra. (b) Same as Fig. 2(a) but for the magnetic $L$ (red) and $R$ (blue) fluctuation spectra.

Consequently, the magnetic energy is dominated by $R$ fluctuations, whereas the kinetic energy is dominated by $L$ fluctuations. The velocity field follows a Kolmogorov $k_{\perp}^{-5 / 3}$ spectrum, while the magnetic spectrum presents a knee around $k_{\perp} d_{i} \sim 10$, with a change in slope going from approximately $k_{\perp}^{-2.8}$ to the WTT prediction $k_{\perp}^{-5 / 2}$.

Figure 3 (top-left panel) displays the isocontours of the bidimensional $L$-kinetic energy spectrum $E_{L}^{u}\left(k_{\perp}, k_{\|}\right)$corresponding to run I. At large scales $(k<50)$, the isocontours are elongated along the $k_{\perp}$ direction, which can be interpreted as a direct consequence of the weak ion-cyclotron wave dynamics. At smaller scales, one can observe a progressive stretching of the isocontours in the $k_{\|}$direction, which is due to the transition toward strong ion-cyclotron wave turbulence, as expected from the spectral properties of the $\chi_{L}^{u}$ parameter (see Fig. 1). Figure 3 (top-right panel) displays the isocontours of the bidimensional $R$-magnetic $E_{R}^{b}\left(k_{\perp}, k_{\|}\right)$energy spectrum for the same simulation.

Interestingly, one can observe the presence of two lobes: one with a strong anisotropy in the $k_{\perp}$ direction and another that extends in the $k_{\|}$direction, showing a propensity toward isotropization as $k_{\|}$increases. This latter property is similar to the one observed for the bidimensional $L$-velocity energy spectrum (Fig. 3, top-left panel) and suggests a possible coupling between ion-cyclotron and whistler waves. The difference in anisotropy between the velocity and magnetic field is clearly visible in real space as can be seen in Fig. 4. We clearly see that the amplitudes of the current density fluctuations are more elongated along the vertical (i.e., $\mathbf{b}_{0}$ ) direction than the vorticity. Several direct numerical experiments provide convincing evidence that electron MHD (EMHD) turbulence develops a strong anisotropy in the presence of a mean magnetic field [39-42]. Yet, EMHD corresponds to Hall MHD with $\mathbf{u}=\mathbf{0}$; the difference between run I and those previously cited may therefore be due to the velocity dynamics. Note, however, that despite the fact that anisotropy of EMHD turbulence is usually considered to be granted and turns out to be mandatory to justify the use of gyrokinetics to model solar wind turbulence $[23,43]$, resonant three-wave interactions of whistler waves allow energy transfer along the external magnetic field direction. These properties are in contrast with resonant interactions of Alfvén waves, which foliate the wave-vector space, a property that strictly forbids any parallel cascade. In weak EMHD turbulence, the transfer along the mean field direction is small only if local interactions in $k_{\perp}$ are dominant [44]. In this case, only counterpropagating whistler waves contribute significantly to the nonlinear dynamics [45], and small scales are preferentially generated perpendicular to the external magnetic field. The presence of the lobe of energy in the parallel direction may therefore also be due to nonlocal interactions.

A straightforward way to discriminate these two scenarios (nonlocal versus ion-cyclotron/whistler interactions) is to perform a numerical experiment with a hyperviscosity large enough to act at large scales. A priori, in this circumstance, the $L$ fluctuations cannot develop their own nonlinear dynamics. Run II described in Table I corresponds precisely to this situation. Figure 3 (bottomright panel) displays the isocontours of the bidimensional $R$-magnetic energy spectrum for this simulation. Clearly, the extent of the lobe in the $k_{\|}$direction is greatly reduced, which strongly suggests that the anomalous spectrum observed in run $\mathrm{I}$ is due to a cross-coupling between ion-cyclotron and whistler waves. The isocontours of the bidimensional $L$-velocity energy spectrum displayed in Fig. 3 (bottom-left panel) are homothetic to the $R$-magnetic one under a $\xi_{-}^{+}$transformation (see Appendix B). Interestingly, the velocity spectrum extends to scales much 

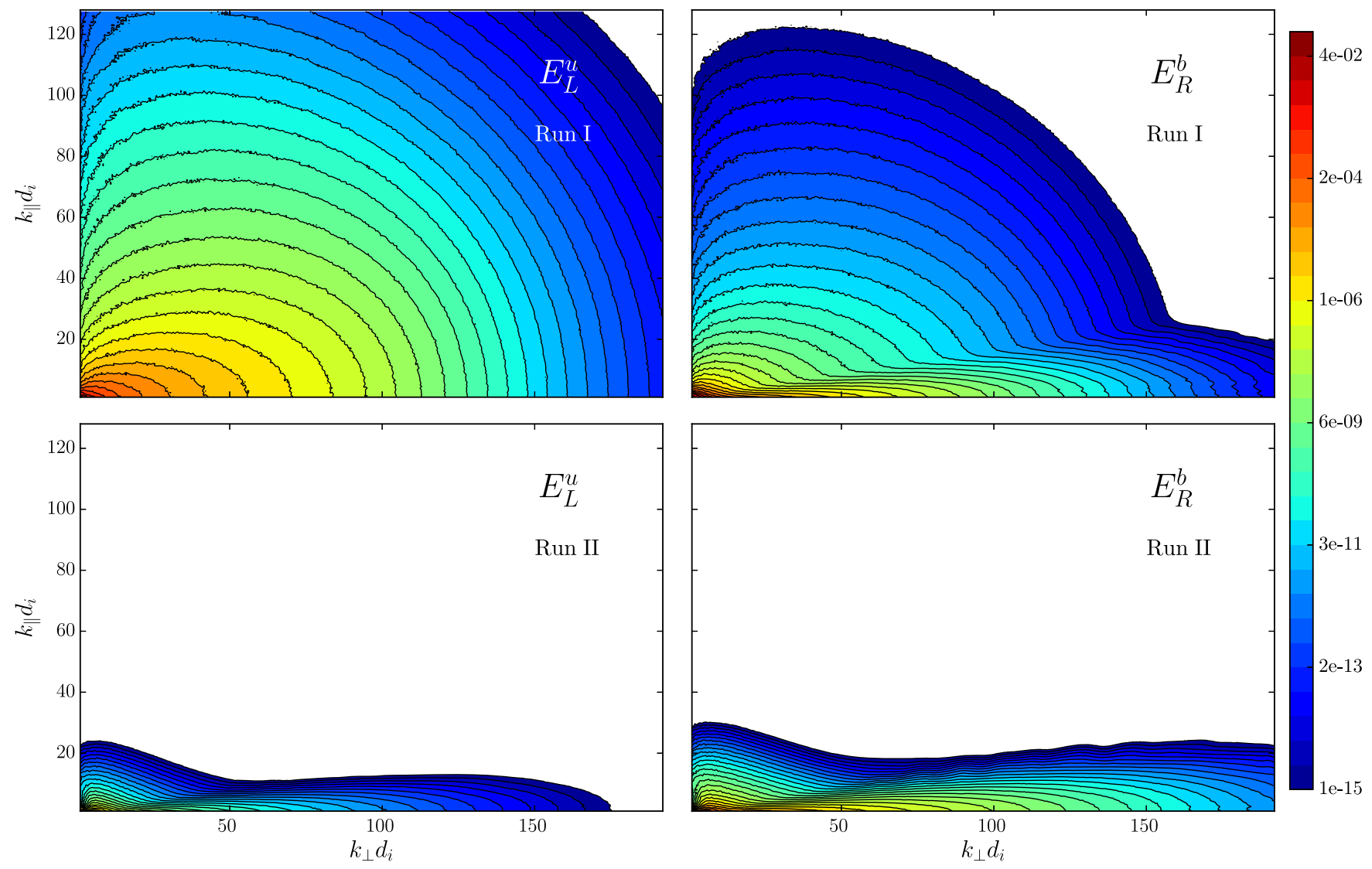

FIG. 3. Iso-contours (in logarithmic scale) of the bi-dimensional $L$ velocity energy spectrum $E_{L}^{u}\left(k_{\perp}, k_{\|}\right)$(left) and $R$ magnetic energy spectrum $E_{R}^{b}\left(k_{\perp}, k_{\|}\right)$(right) corresponding to Run I (top) and II (bottom).

smaller than the Kolmogorov dissipation microscale. This property reflects the fact that the velocity is enslaved to the magnetic field via the Lorentz force term $\mathbf{j} \times \mathbf{b}$, which is not directly affected by the velocity hyperdiffusivity. However, the presence of a small lobe in the $k_{\|}$shows a small backreaction of the velocity via the nonlinear advection term $\mathbf{u} \cdot \nabla \mathbf{u}$. This demonstrates that, contrary to the common belief, EMHD is not simply the small-scale limit $k d_{i} \gg 1$ of Hall MHD and is independent of the Prandtl number [46].

\section{Shell-to-shell energy transfer functions}

To further investigate the cross-coupling between ioncyclotron and whistler waves, we have performed a detailed analysis of the shell-to-shell energy transfer functions. The shell-to-shell kinetic and magnetic energy transfer functions are defined by [47]

$$
\begin{gathered}
\frac{\partial E^{u}(\mathbf{k})}{\partial t}=\sum_{\mathbf{p}}\left[T_{u u}^{u}(\mathbf{k}, \mathbf{p})-T_{b b}^{u}(\mathbf{k}, \mathbf{p})\right], \\
\frac{\partial E^{b}(\mathbf{k})}{\partial t}=\sum_{\mathbf{p}}\left[T_{b u}^{b}(\mathbf{k}, \mathbf{p})-T_{u b}^{b}(\mathbf{k}, \mathbf{p})-d_{i} T_{b j}^{b}(\mathbf{k}, \mathbf{p})\right],
\end{gathered}
$$

where

$T_{Y Z}^{X}(\mathbf{k}, \mathbf{p})=\sum_{\mathbf{q}} \operatorname{Im}\left\{[\mathbf{k} \cdot \hat{\mathbf{Z}}(\mathbf{p})]\left[\hat{\mathbf{Y}}(\mathbf{q}) \cdot \hat{\mathbf{X}}^{*}(\mathbf{k})\right]\right\} \delta_{\mathbf{q}+\mathbf{p}, \mathbf{k}}$

is the transfer function to the mode $\mathbf{k}$ of field $\mathbf{X}$ from mode $\mathbf{p}$ of field $\mathbf{Z}$, mediated by all possible triadic interactions with modes $\mathbf{q}$ of fields $\mathbf{Y}$ that respect the condition $\mathbf{k}=\mathbf{p}+\mathbf{q}$. Im denotes the imaginary part, and the asterisk is the complex conjugate. Note that, for the sake of clarity, we omit the hyperdissipative $T_{\text {diss }}^{u}=2 \nu_{3} k^{6} E^{u}(\mathbf{k})$ and hyperdiffusive $T_{\text {diss }}^{b}=2 \eta_{3} k^{6} E^{b}(\mathbf{k})$ terms as well as the forcing terms. The energy flux flowing toward a given $k$ scale via the $T_{Y Z}^{X}$ channel is given by

$$
\Pi_{Y Z}^{X}(\mathbf{k})=\sum_{\mathbf{k}^{\prime}=0}^{\mathbf{k}} \sum_{\mathbf{p}} T_{Y Z}^{X}\left(\mathbf{k}^{\prime}, \mathbf{p}\right) .
$$

To study the perpendicular cascade, we consider concentric cylindrical shells along $\mathbf{b}_{0}$ with constant width on a logarithmic scale, which we define as the region $k_{0} 2^{n / 4} \leq$ $k_{\perp} d_{i} \leq k_{0} 2^{(n+1) / 4}$ for the shells numbered $4 \leq n \leq N$, where we set $k_{0}=2$ and $N=25$. A schematic representation of the various energy fluxes that we may find a priori 


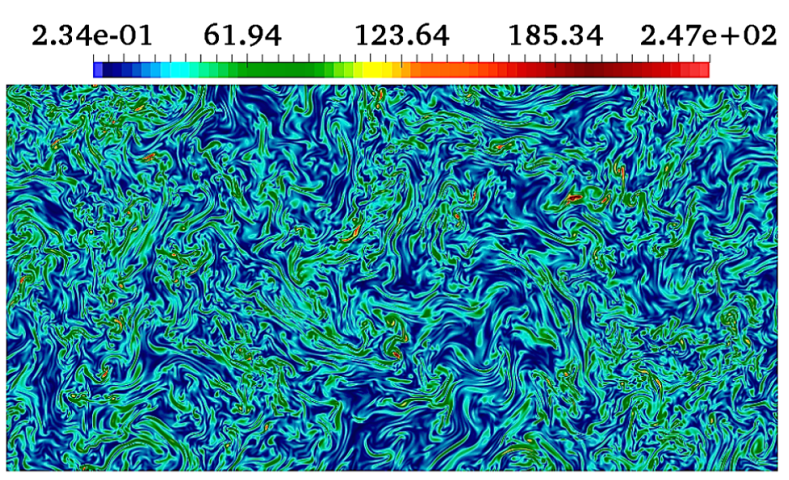

(a)

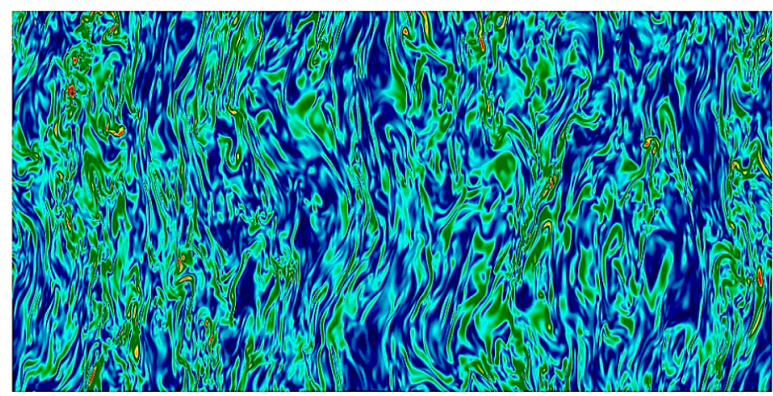

(c)

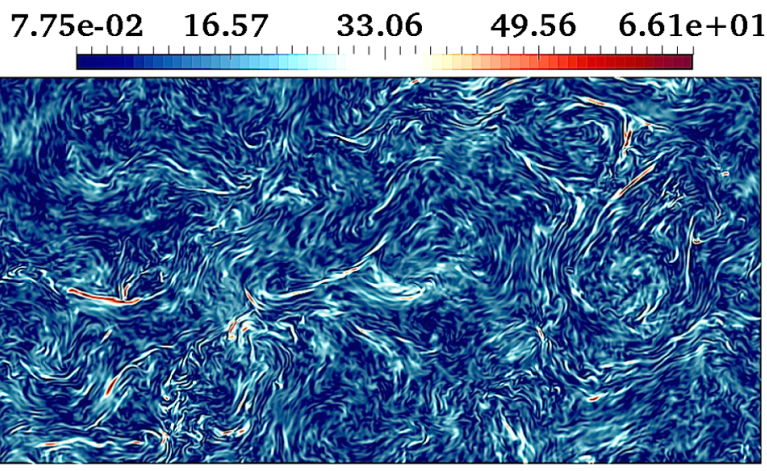

(b)

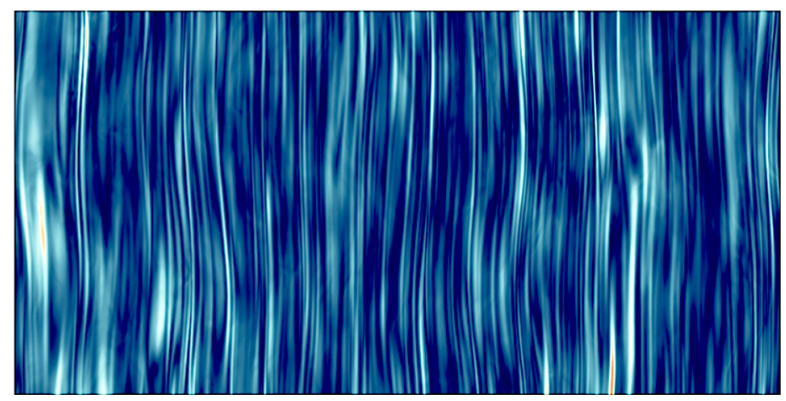

(d)

FIG. 4. Amplitude of vorticity (left panels) and current density (right panels) fluctuations in a field-perpendicular (top panels) and field-parallel (bottom panels) cross section of the simulation domain (run I). Clearly, the anisotropy is stronger for the current density than the vorticity fluctuations. The width of the snapshots is equal to $2 \times d_{i}$ and the height to $1 \times d_{i}$.

in the inertial range of Hall MHD turbulence is given in Fig. 5. Since the kinetic and magnetic energies are not inviscid and ideal invariants, nontrivial energy fluxes may also exist between them at a given scale.

The various energy fluxes normalized by the total energy flux at each scale are displayed in Fig. 6 (run I). It shows the relative proportion of energy flowing through the velocity and magnetic nonlinear channels. Despite the fact

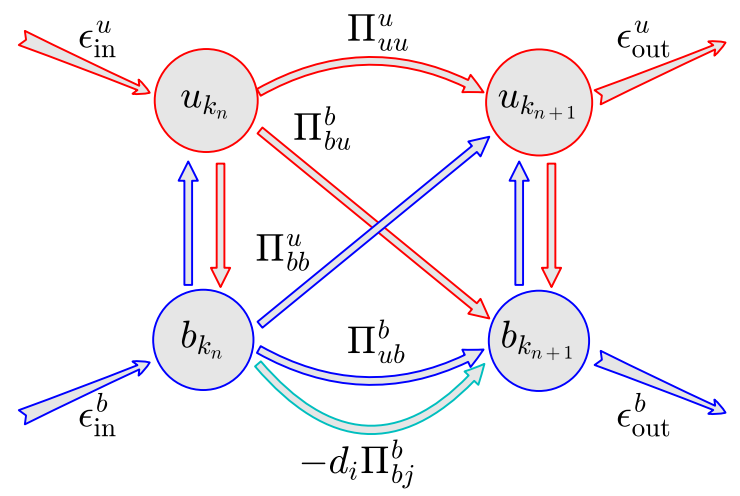

FIG. 5. Schematic representation of various energy fluxes at a given scale in the inertial range of Hall MHD turbulence. Note that because kinetic and magnetic energies are not conserved separately, nontrivial energy fluxes inside the same wave-vector domain may exist (vertical arrows). The arrows labeled $\epsilon_{\text {in,out }}^{u, b}$ represent the different incoming or outgoing energy fluxes. that the same amount of kinetic and magnetic energy is injected in the system, one can see that the velocity flux $\Pi_{u и}^{u}$ channel carries about 3 times more energy than the Hall $-d_{i} \Pi_{b j}^{b}$ one. Consequently, the dissipation rate of kinetic energy $\left[\Pi_{\text {diss }}^{u}(\mathbf{k})=2 \nu_{3} \sum_{\mathbf{k}^{\prime}=0}^{\mathbf{k}^{\prime}=\mathbf{k}} \mathbf{k}^{\prime 6} E^{u}\left(\mathbf{k}^{\prime}\right)\right]$ is about 3 times higher than the dissipation rate of magnetic energy $\left[\Pi_{\text {diss }}^{b}(\mathbf{k})=2 \eta_{3} \sum_{\mathbf{k}^{\prime}=0}^{\mathbf{k}^{\prime}=\mathbf{k}} \mathbf{k}^{\prime 6} E^{b}\left(\mathbf{k}^{\prime}\right)\right]$. Interestingly, in the absence of a mean magnetic field, kinetic and magnetic energy fluxes are in equipartition (see Ref. [35]). In other

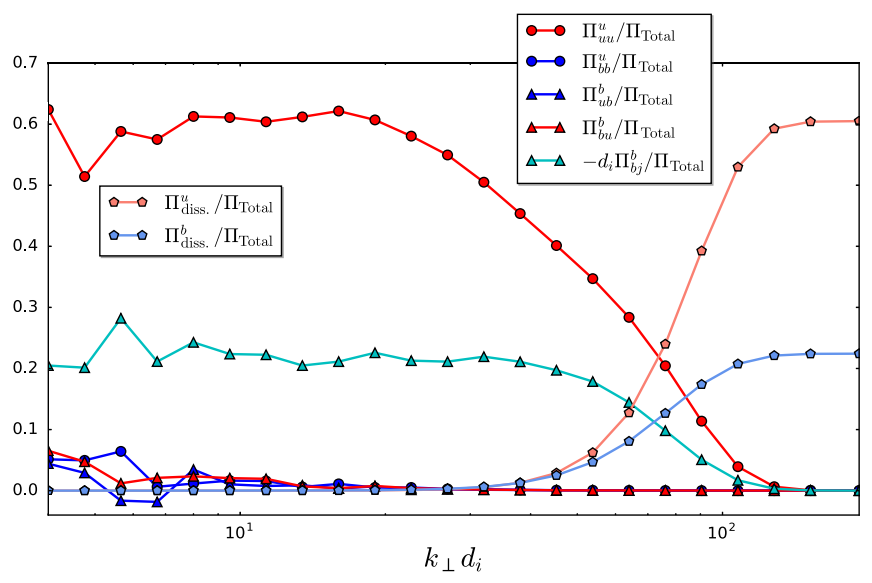

FIG. 6. Normalized energy fluxes versus $k_{\perp} d_{i}$ (run I). 
words, the relative proportion of kinetic and magnetic energy transfer rates is directly proportional to the strength of the mean magnetic field. This suggests that the energy tends to follow the nonlinear path that shows the least resistance (i.e., for which the nonlinear timescale is the smallest), which is somewhat like the way an electric current is distributed in a circuit with different resistivity.

The kinetic and magnetic energy transfer functions defined in Eqs. (11)-(13) normalized, respectively, by the total kinetic and magnetic energy transfer functions are displayed in Fig. 7. Clearly, the Hall term is the dominant nonlinear channel to cascade the magnetic energy toward small scales. The kinetic energy, on the other hand, cascades predominately via the advection term $(\mathbf{u} \cdot \nabla) \mathbf{u}$. The latter property, given that the $L$ fluctuations do not belong to the weak turbulent regime for all $k_{\perp}$, may explain the $k_{\perp}^{-5 / 3}$ spectrum observed for the $L$-fluctuation velocity [see Fig. 2(a)]. The signals relative to $-d_{i} T_{b j}^{b}$ and $T_{u u}^{u}$ being concentrated around the diagonal $k_{\perp}=p_{\perp}$ means that direct and local energy transfers dominate. We can therefore confirm that the nonlocality of the energy cascade is not responsible for the anomalous anisotropy observed in our simulations (see discussion in Sec. III C). The cross transfers of energy from kinetic to magnetic fields $T_{b u}^{b}$, as well as from magnetic to kinetic fields $T_{b b}^{u}$ mediated, respectively, by the nonlinear terms $(\mathbf{b} \cdot \nabla) \mathbf{u}$ and $(\mathbf{b} \cdot \nabla) \mathbf{b}$, become negligible for scales $k_{\perp} d_{i}>10$. Remarkably, this critical scale corresponds precisely to the one for which we observed a knee in the $R$-magnetic energy spectra and is close to the critical scale corresponding to the transition from weak ion-cyclotron wave turbulence to strong ioncyclotron wave turbulence. This suggests that the anomalous spectrum in $k_{\perp}^{-2.8}$ observed at $k_{\perp} d_{i}<10$ is due to the influence of the ion-cyclotron dynamics, whereas the subsequent $k_{\perp}^{-2.5}$ spectrum may correspond to a pure weak whistler wave turbulence regime. Although this conclusion may seem appealing, it remains to show that resonant whistler three-wave interaction processes, the "atom" of the statistical WTT, are effectively at work. This is absolutely not guaranteed given that the whistler waves are embedded in a sea of highly nonlinear ion-cyclotron fluctuations. The purpose of Sec. VI is to precisely address this delicate issue.

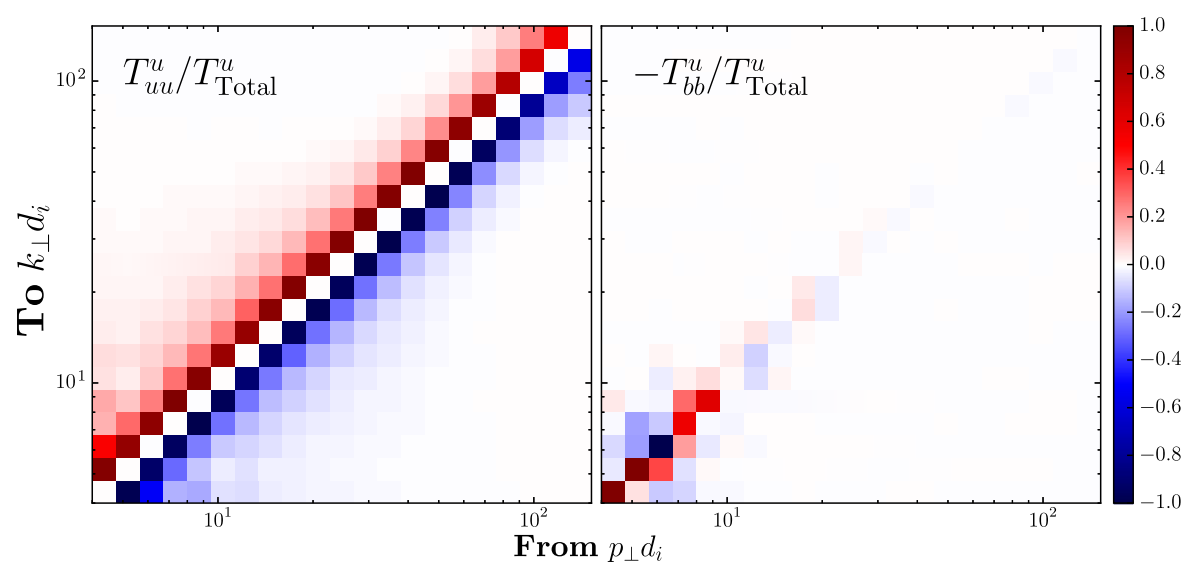

(a)
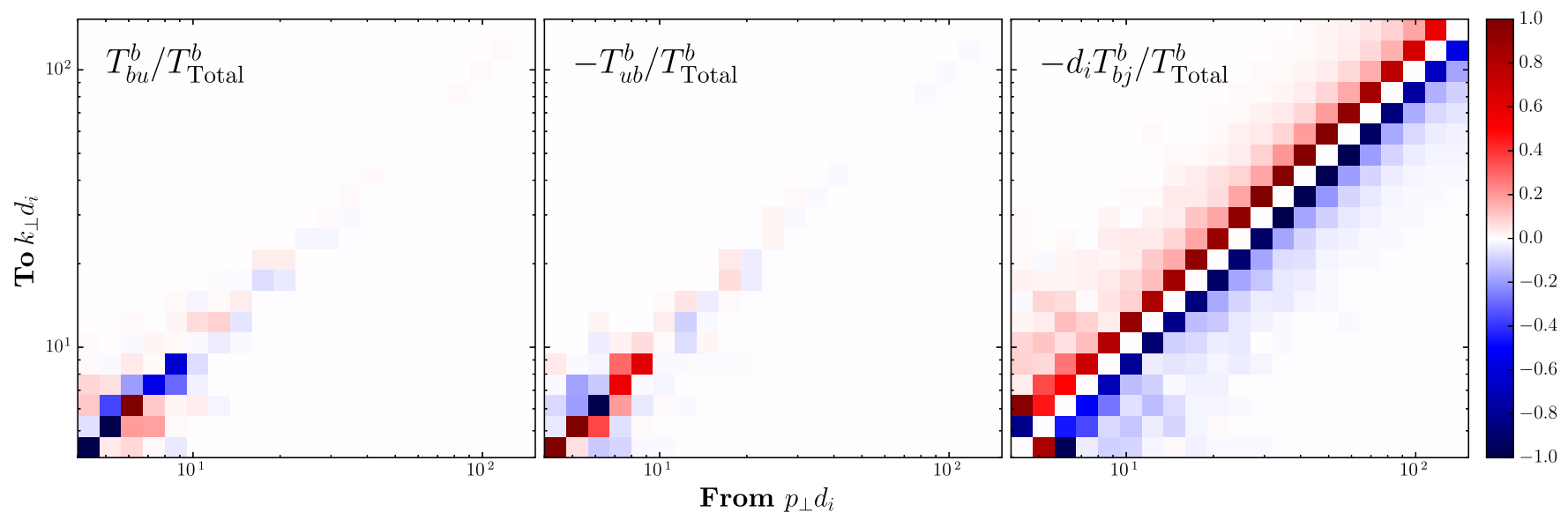

(b)

FIG. 7. Normalized transfer functions for the kinetic energy (top) and magnetic energy (bottom) for run I. 


\section{MEASUREMENTS AND CHARACTERIZATION OF THE RESONANT NONLINEAR WAVE INTERACTIONS}

Even though the Hall MHD fluid equations are deterministic, turbulence is fundamentally a chaotic motion. Consequently, only averaged quantities are experimentally reproducible and can be studied thoroughly. However, by adopting a statistical description, the cost is high as we have to handle an infinite system of equations. In WTT, the infinite hierarchy of equations is closed because, in the long-time limit, the nonlinear regeneration of third-order moments depends essentially on products of second-order moments and not on the fourth-order cumulant, which is not a resonant term [48]. Consequently, the nonlinear energy transfer in WTT involves mainly resonant threewave interaction processes. Henceforth, it appears essential to check whether such a process is effectively at work in our simulations before using the theoretical WTT framework. To do so, it is necessary to use higher-order polyspectra, which can be seen as a generalization of the Fourier analysis to include information about phase coherence.

\section{A. Definition of bispectra and physical interpretation}

A linear system can be described by a superposition of statistically independent Fourier modes, and all the relevant information is contained in the power spectral density (or the autocorrelation function). However, if some nonlinear physical processes exist, then the phases of the Fourier modes are not independent anymore, and information is also conveyed by the phases. By construction, second-order statistics are phase blind, and information about phase must be recovered from higher-order polyspectra. The use of higher-order moments nullifies all Gaussian random effects of the process, and the bispectrum can then quantify the degree of the remaining nonlinear coupling. The bispectrum is defined by

$$
B\left(\omega_{1}, \omega_{2}\right)=\left\langle a\left(\omega_{1}\right) a\left(\omega_{2}\right) a\left(\omega_{1}+\omega_{2}\right)^{*}\right\rangle,
$$

where the average $\langle\ldots\rangle$ stands for averaging over time windows and $a$ represents the Fourier transform in time of the physical quantity of interest. The bispectrum measures the amount of phase coherence between three Fourier modes that obey the frequency summation rule $\omega_{1}+\omega_{2}=\omega_{3}$; it can be seen as the frequency domain representation of the third-order cumulant, the building block of WWT. The bicoherence

$$
C^{2}\left(\omega_{1}, \omega_{2}\right)=\frac{\left|\left\langle a\left(\omega_{1}\right) a\left(\omega_{2}\right) a\left(\omega_{1}+\omega_{2}\right)^{*}\right\rangle\right|^{2}}{\left\langle\left|a\left(\omega_{1}\right) a\left(\omega_{2}\right)\right|^{2}\right\rangle\left\langle\left|a\left(\omega_{1}+\omega_{2}\right)^{*}\right|^{2}\right\rangle}
$$

removes the magnitude dependence of the bispectrum; it is the normalized representation. With such a normalization, the coherence lies between 0 (no correlation) and 1 (perfect correlation). If the three Fourier components $a\left(\omega_{1}\right), a\left(\omega_{2}\right)$, and $a\left(\omega_{1}+\omega_{2}\right)^{*}$ are phase locked, they will sum without canceling, resulting in a large value of the bicoherence even though each phase, when taken separately, may vary in a random way. Therefore, the bicoherence measures the proportion of the signal energy at any bifrequency $\left(\omega_{1}, \omega_{2}\right)$ that is quadratically phase coupled to $\omega_{3}=\omega_{1}+\omega_{2}$. Note that it is necessary to have a large time separation between the phases' and the amplitudes' characteristic times to produce a significant bicoherence. The use of bicoherence is therefore particularly relevant for wave phenomena with a weak departure from nonlinearity and is a natural statistical tool to test the adequacy of weak interaction theory as a description of the nonlinear coupling. Bicoherence has been widely used to examine various physical systems, including space plasma physics [49,50], plasma fusion devices [51,52], ocean waves [53], weak turbulence of gravity-capillary waves [9], or cosmology [54], to mention just a few. For the sake of clarity, we have defined bicoherence by considering a single quantity $a$, but bicoherence can easily be extended to study the phase coupling between different variables. To distinguish the two situations, one should use the prefix "auto" for a single quantity and "cross" for multiple quantities.

\section{B. Experimental setup}

The bicoherence is related to the shape (in a statistical sense) of the time series. For a finite time series, even a truly Gaussian process will have a nonzero bicoherence. To decrease the noise level under the physically pertinent signal, it is therefore necessary to consider a large statistical ensemble and thus integrate the Hall MHD equations over a long time period. This turns out to be prohibitory for the first numerical experiment (run I). We therefore consider a numerical simulation with a smaller resolution (run III) of $N_{\perp}^{2} \times N_{\|}=128^{2} \times 64$ collocation points. The hyperdiffusivity and hyperviscosity are adjusted consequently with values of $\eta_{3}=3.2 \times 10^{-8}$ and $\nu_{3}=4.25 \times 10^{-9}$, respectively. All other parameters are otherwise identical to those of run I. We have checked (not shown) that this numerical experiment qualitatively presents a similar "anomaly" at the level of the anisotropy and the power spectral index. However, because of the reduced spectral resolution, the wave-number extension where highly nonlinear left-handed fluctuation are observed is reduced compared to run I.

We record a time interval of the generalized Elsässer fields $\mathcal{Z}_{\Lambda}^{s}(x, t)$ from a real space Eulerian probe. The modes $|k| \in\left[0,4 d_{i}\right]$ are filtered so as to avoid any integral scale effect. We consider a time interval of $6900 \omega_{c i}$ with a sampling frequency of $\delta t \sim 0.014 \omega_{c i}$. From $\mathcal{Z}_{\Lambda}^{s}(x, t)$, we compute the Fourier transform in time over 100 time windows to obtain $\mathcal{Z}_{\Lambda}^{s}(x, \omega)$. Before taking the Fourier transform, each sample is multiplied by a Hamming window and detrended using a standard linear least-squares method. Cross-bicoherence is then computed from 
Eq. (16). Furthermore, we calculate a fiducial bicoherence from 100 synthetic signals $f(t)=\sum_{i=1}^{3} \sin \left(\left(\omega_{i}+\delta \omega_{i}\right) t+\right.$ $\left.\phi_{i}\right)+\delta a t$ with similar sampling frequency and where $\omega_{1}=10.4 \omega_{c i}, \quad \omega_{2}=2.5 \omega_{c i}$, and $\omega_{3}=\omega_{1}+\omega_{2}$. The phases $\phi_{i}$ are randomly distributed over each time window such that $\phi_{i} \in[-\pi, \pi]$ and $\phi_{3}=\phi_{1}+\phi_{2}$. Note that $\delta \omega_{i}$ are random numbers modeling artificial nonlinear frequency broadening with $\delta \omega_{i} \in[-0.001,0.001]$. Finally, $\delta a$ simulates a Gaussian noise at the level of the amplitude $(\delta a \in[-0.01,0.01])$. This fiducial bicoherence gives an idea of the statistical noise inherent to the statistical ensemble that we consider, as well as the signature of quadratically phase-coupled waves.

\section{Results}

Figure 8 displays the temporal evolution of right-handed $\mathcal{Z}_{+}^{+}(x, t)$ and left-handed $\mathcal{Z}_{+}^{-}(x, t)$ fluctuations, as well as the synthetic fiducial signal over one of the 100 windows. Clearly, the $R$ fluctuations evolve on shorter timescales than the $L$ fluctuations. Interestingly, the latter displays a periodicity of about $3 \omega_{c i}^{-1}$, but shorter characteristic times are also excited. The use of bicoherence will show whether these short time fluctuations result from the coupling with whistler waves or are the consequence of nonlinear processes involving exclusively ion-cyclotrontype fluctuations.

Figure 9(a) displays the cross-bicoherence

$$
C_{R R}^{2}\left(\omega_{1}, \omega_{2}\right)=\frac{\left|\left\langle\mathcal{Z}_{+}^{+}\left(\omega_{1}\right) \mathcal{Z}_{-}^{-}\left(\omega_{2}\right) \mathcal{Z}_{+}^{+}\left(\omega_{1}+\omega_{2}\right)^{*}\right\rangle\right|^{2}}{\left\langle\left|\mathcal{Z}_{+}^{+}\left(\omega_{1}\right) \mathcal{Z}_{-}^{-}\left(\omega_{2}\right)\right|^{2}\right\rangle\left\langle\left|\mathcal{Z}_{-}^{-}\left(\omega_{1}+\omega_{2}\right)^{*}\right|^{2}\right\rangle}
$$

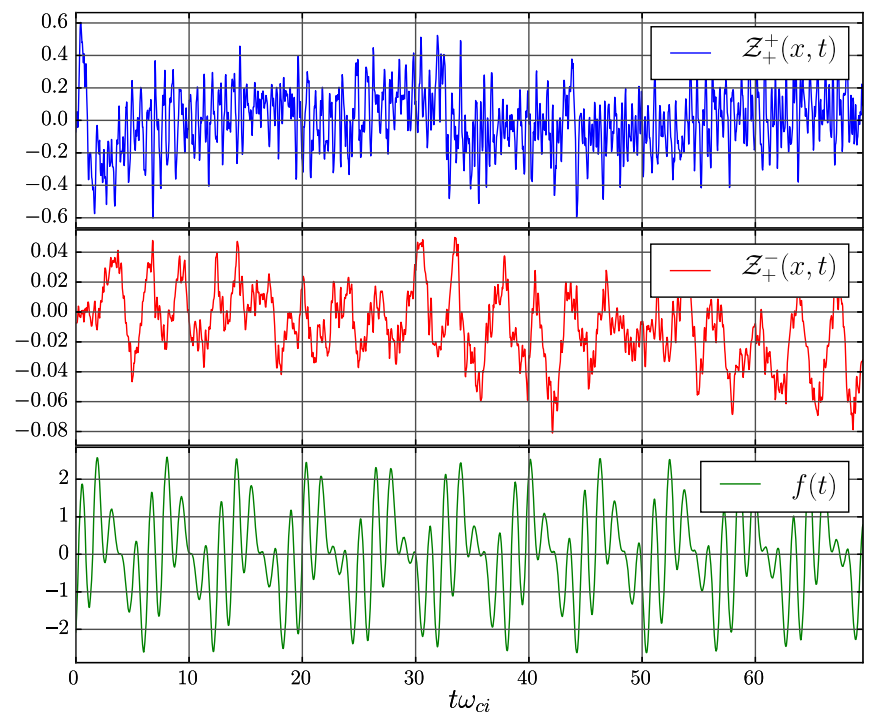

FIG. 8. Temporal evolution of right-handed $\mathcal{Z}_{+}^{+}(x, t)$ (top) and left-handed $\mathcal{Z}_{+}^{-}(x, t)$ (middle) fluctuations, as well as the synthetic fiducial signal (bottom; see text) over one of the 100 windows (run III). resulting from the coupling between three whistler waves, two of which have opposite. Figure 9(b) displays a similar cross-bicoherence but for ion cylotron waves,

$C_{L L}^{2}\left(\omega_{1}, \omega_{2}\right)=\frac{\left|\left\langle\mathcal{Z}_{+}^{-}\left(\omega_{1}\right) \mathcal{Z}_{-}^{+}\left(\omega_{2}\right) \mathcal{Z}_{+}^{-}\left(\omega_{1}+\omega_{2}\right)^{*}\right\rangle\right|^{2}}{\left\langle\left|\mathcal{Z}_{+}^{-}\left(\omega_{1}\right) \mathcal{Z}_{-}^{+}\left(\omega_{2}\right)\right|^{2}\right\rangle\left\langle\left|\mathcal{Z}_{+}^{-}\left(\omega_{1}+\omega_{2}\right)^{*}\right|^{2}\right\rangle}$

For convenience, the norm of the time Fourier transform of the generalized Elsässer fields relative to one time window normalized by their respective maximum value around $\omega_{c i}=1$ are also shown. The Nyquist theorem restricts the displays of the cross-bicoherence to the triangle defined by $\omega_{1}+\omega_{2} \leq \mathrm{nf}$, where $\mathrm{nf} \sim 15 \omega_{c i}$ is the Nyquist frequency. The circular domain located at the bottom left of the figures corresponds to the fiducial bicoherence described in Sec. IV B.

A significant cross-bicoherence magnitude emerges from the statistical convergence noise at all frequencies, which confirms that three-wave resonant processes are indeed present in the signal. A noticeable organization of the cross-bicoherence is clearly visible. The crossbicoherence level is not homogeneous, showing preferential interaction among the waves. Not surprisingly, the highest value for ion-cyclotron cross-bicoherence $C_{L L}$ is seen for values of $\omega_{1}, \omega_{2}$ such that $\omega_{1}+\omega_{2} \leq 2 \omega_{c i}$ [see inset on Fig. 9(b)]. The fact that significant crossbicoherence is found for $\omega_{1}, \omega_{2} \in\left[\omega_{c i}, 2 \omega_{c i}\right]$ may be attributed to nonlinear frequency broadening. Interestingly, this observation is also valid for whistler cross-bicoherence $C_{R R}$. Conversely, it can be observed in Fig. 9(b) that the ion-cyclotron cross-bicoherence $C_{L L}$ above the statistical noise is present at frequency $\omega>\omega_{c i}$ far from the linear frequency asymptote. This suggests that ion-cyclotron and whistler fluctuations are nonlinearly intricate. To test this idea, we compute the cross-bicoherence $C_{L R}$ resulting from the coupling between an ion-cyclotron wave and two counterpropagating whistler waves with

$$
C_{L R}^{2}\left(\omega_{1}, \omega_{2}\right)=\frac{\left|\left\langle\mathcal{Z}_{-}^{+}\left(\omega_{1}\right) \mathcal{Z}_{+}^{+}\left(\omega_{2}\right) \mathcal{Z}_{-}^{-}\left(\omega_{1}+\omega_{2}\right)^{*}\right\rangle\right|^{2}}{\left\langle\left|\mathcal{Z}_{-}^{+}\left(\omega_{1}\right) \mathcal{Z}_{+}^{+}\left(\omega_{2}\right)\right|^{2}\right\rangle\left\langle\left|\mathcal{Z}_{-}^{-}\left(\omega_{1}+\omega_{2}\right)^{*}\right|^{2}\right\rangle} .
$$

Figure 10 displays such a cross-bicoherence and confirms the result obtained from the detailed study of the transfer functions provided in Sec. III D. Ion-cyclotron and whistler waves are indeed nonlinearly entangled. Note that all the different combinations of cross-bicoherence are qualitatively similar (not shown). However, we observe that the cross-bicoherence involving at least two counterpropagating waves is larger than the corresponding crossbicoherence for which only waves propagating in the same direction take part. 


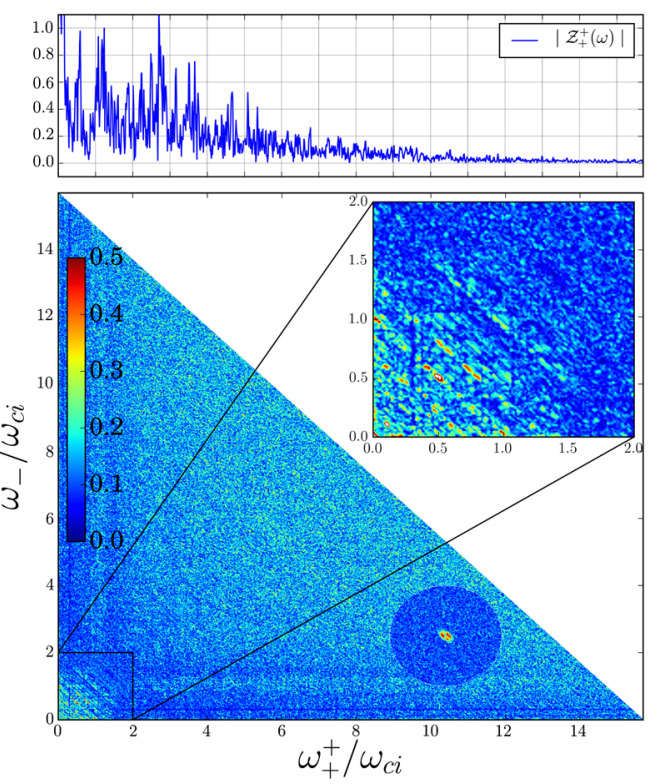

(a)
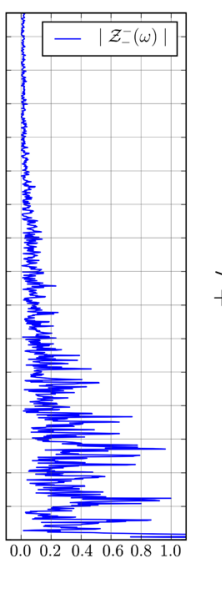
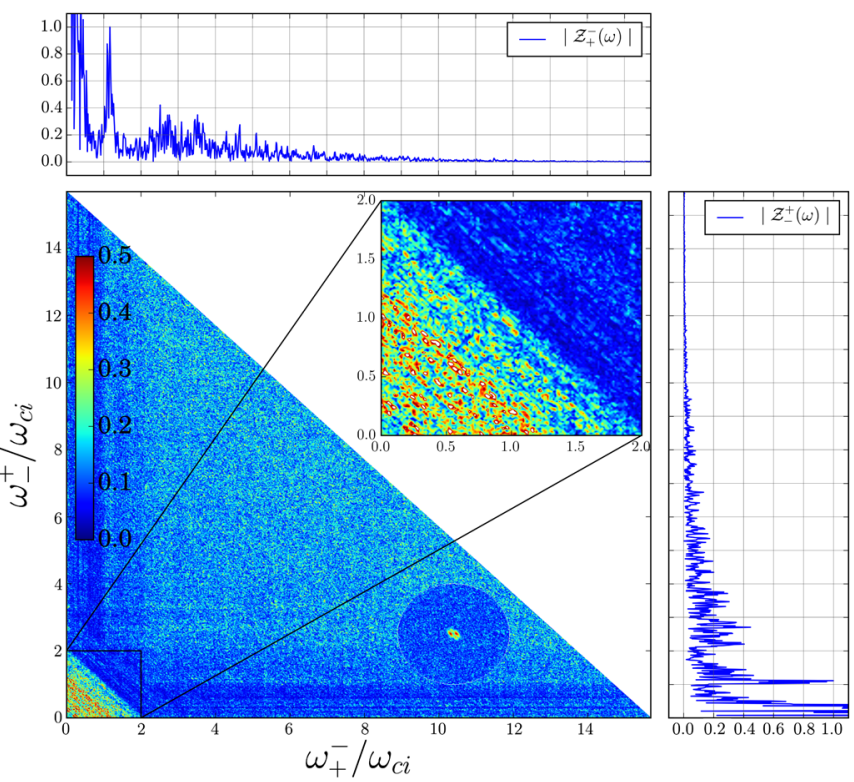

(b)

FIG. 9. (a) Cross-bicoherence $C_{R R}$ resulting from the coupling between three whistler waves, two of which have opposite polarity. (b) Cross-bicoherence $C_{L L}$ resulting from the coupling between three ion-cyclotron waves, two of which have opposite polarity. For convenience, the norm of the time Fourier transform of the generalized Elsässer fields normalized by their respective maximum value around $\omega_{c i}=1$ are also shown above and beside each plot. The intense signal surrounded by a circle of weaker signal corresponds to the fiducial bicoherence mentioned in the text. This gives an idea of the statistical noise inherent to the statistical ensemble that we consider, as well as the signature of quadratically phase-coupled waves.

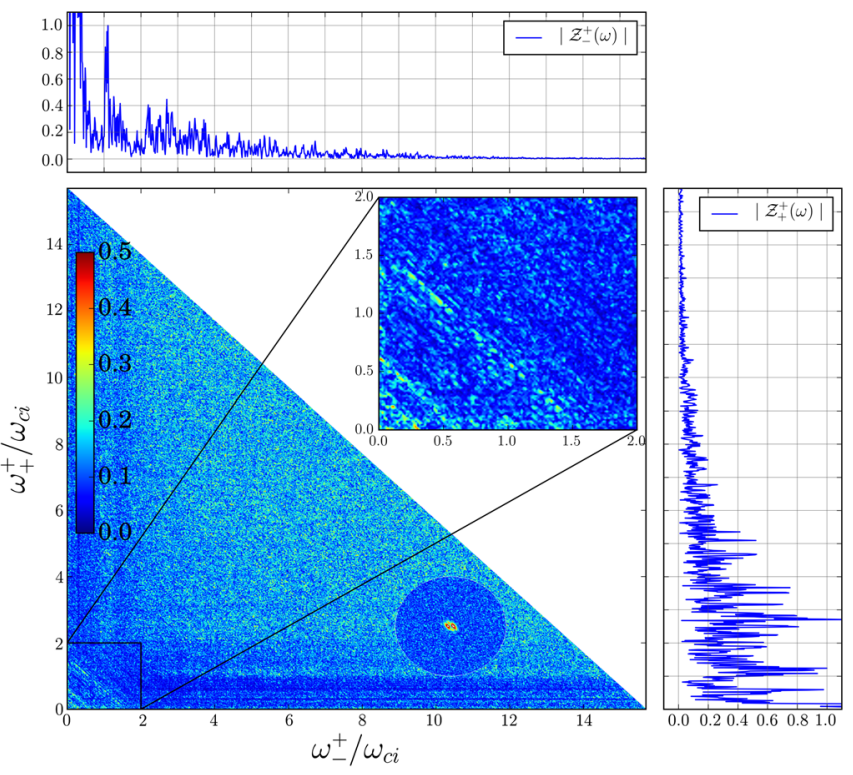

FIG. 10. Cross-bicoherence $C_{L R}$ resulting from the coupling between an ion-cyclotron wave and two counterpropagating whistler waves. For convenience, the modulus of the time Fourier transform of the generalized Elsässer fields normalized by their respective maximum value around $\omega_{c i}=1$ are also shown above and beside the plot. The intense signal surrounded by a circle of weaker signal corresponds to the fiducial bicoherence mentioned in the text. This gives an idea of the statistical noise inherent to the statistical ensemble that we consider, as well as the signature of quadratically phase-coupled waves.
The new and important information that we can extract from the cross-bicoherence analysis is that the resonant triadic interactions survive the highly nonlinear bath of ion-cyclotron fluctuations. However, this coupling is significantly higher in the frequency domain where only weakly nonlinear waves coexist, namely, for $\omega_{1}, \omega_{2}$ such that $\omega_{1}+\omega_{2} \leq 2 \omega_{c i}$. This observation suggests an alteration of the resonant triadic interactions by the presence of the strong wave ion-cyclotron turbulence. In order to study the nonlinear dynamics in more depth, in the following section, we focus on the properties of the space-time Fourier spectrum. Such a technique allows the precise identification and extraction of the waves and nonlinear structures contributions to the total energy.

\section{SPACE-TIME FOURIER SPECTRA}

\section{A. Experimental setup}

Computation of the space-time Fourier $(\mathbf{k}-\omega)$ spectrum requires simultaneous space and time Fourier transforms. The frequency sampling must be at least 2 times larger than the frequency of the fastest waves of the system, and the total time of acquisition should be larger than both the period of the slowest waves and the turnover time of the slowest eddies. These constraints turn out to be numerically redhibitory for run $\mathrm{I}$. We thus consider a numerical simulation with a smaller resolution (run IV) 
of $N_{\perp}^{2} \times N_{\|}=256^{2} \times 128$ collocation points. The hyperdiffusivity and hyperviscosity are adjusted consequently with values of, respectively, $\eta_{3}=4.5 \times 10^{-10}$ and $\nu_{3}=$ $7.6 \times 10^{-11}$. All other parameters are otherwise identical to those of run I. We have checked (not shown) that this numerical experiment qualitatively presents a similar "anomaly" at the level of the anisotropy and the power spectral index. However, because of the reduced spectral resolution, the wave-number extension where highly nonlinear left-handed fluctuations are observed is reduced compared to run I. Because the dispersion relation of incompressible Hall MHD depends on the angle $\theta=$ $\arccos \left(k_{z} / \mathbf{k}\right)$ of the wave number relative to the mean field (see Appendix A 2), we consider the Fourier transform of the generalized Elsässer fields along rays of wave numbers at constant $\theta$. We consider five different angles $\theta_{i}$ such that their cosines are equal to $0.1,0.3,0.5,0.7,0.9$. For each of these five angles, we consider 10 rays at constant $\varphi$, where $\varphi=\arctan \left(k_{y} / \mathbf{k}\right)$ is the angle between the wave vector and the direction $y$ perpendicular to the mean field. We take $\varphi_{i}=-\pi / 2+i \times \pi / 10$ for $i \in \mathbb{N} \mid i \in$ $[0,9]$. Negative values of the wave number $k_{x}$ are not considered because of the symmetry of the Fourier representation of real fields $\left[u(-k)=u^{*}(k)\right]$. Along each of these $10 \times 5$ wave-vector rays, we record time evolution of the generalized Elsässer fields on 64 points uniformly distributed $\left(k_{j}=j \times d_{i}|j \in \mathbb{N}| j \in[1,64]\right)$. Because these wave numbers do not match the grid points, such a procedure requires 3D Fourier space interpolation. We have opted for a cubic spline technique. Because of simulation storage constraints (both in space and in I/O speed), we record only the real part of the $x$ component of the generalized Elsässer field. We have checked that another choice does not qualitatively change the result. We choose an acquisition frequency equal to $d t=4 \times 10^{-3} \omega_{c i}$, which allows us to correctly resolve the fastest waves that we observe in the simulation. The total time of acquisition is equal to $t \sim 60 \omega_{c i}$, which enables us to capture both the ion-cyclotron waves and the slowest eddies. In order to mitigate spectral leakage, we systematically apply a Hamming window and subtract the mean value of the different signals before computing the temporal Fourier transform.

\section{B. Results}

We show in Fig. 11 the space-time Fourier spectrum of the $x$ component of the generalized Elsässer variables $Z_{-}^{+}$ and $Z_{+}^{-}$(ion cyclotron) corresponding to $\cos (\theta)=0.7$. At large scales $\left(k d_{i}<15\right)$, energy is mainly localized on the dispersion relation. At smaller scales, one can observe a significant broadening of the $\left(\omega-k d_{i}\right)$ distribution reflecting the transition toward strong ion-cyclotron wave turbulence. The signal at high frequencies $\left(\omega / \omega_{c i}>1\right)$ and low wave numbers $(k<5)$ matches the linear dispersion

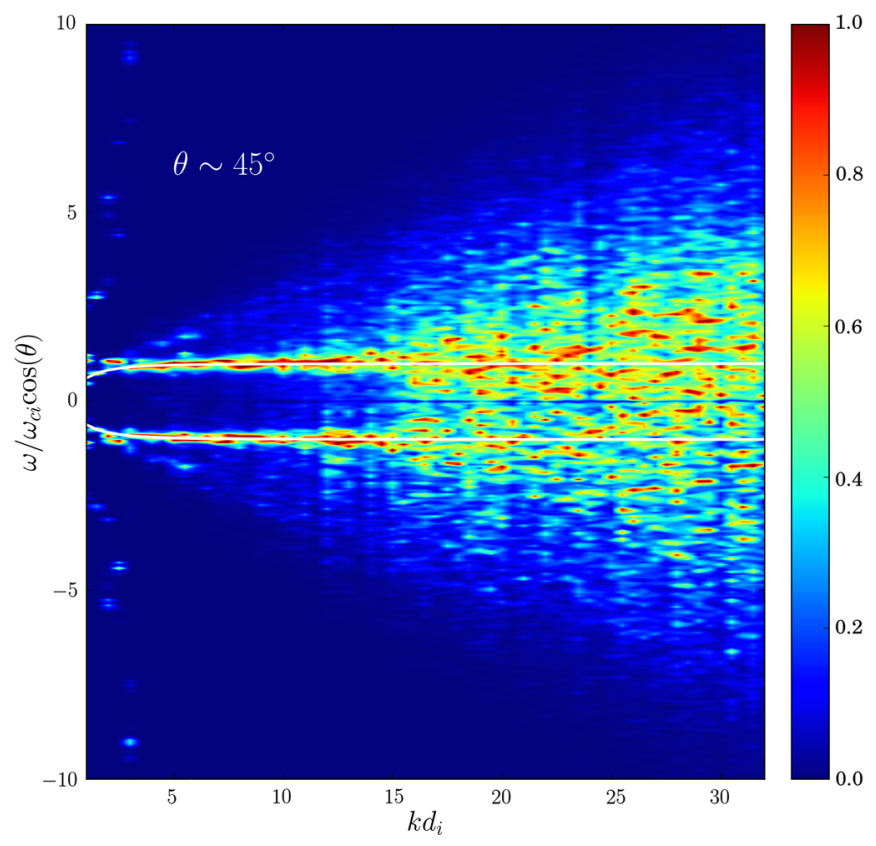

FIG. 11. Space-time Fourier spectrum of the $x$ component of the generalized Elsässer $Z_{-}^{+}$(positive frequency) and $Z_{+}^{-}$ (negative frequency) fields at $\theta \sim 45^{\circ}$. The solid white line is the theoretical ion-cyclotron linear dispersion relation. The color map is normalized to the maximum value of the spectrum at each fixed $k$.

relation of the whistler and is a direct signature of the crosscoupling of the two populations of waves. Figure 12(a) displays the mean of five space-time Fourier spectra of the $x$ component of the generalized Elsässer variables $Z_{+}^{+}$and $Z_{-}^{-}$ corresponding to five different angles $\theta \sim 84^{\circ}, 72^{\circ}, 60^{\circ}, 45^{\circ}$, $25^{\circ}$. Before the mean is taken, each signal is normalized to the maximum value at each fixed $k$. At large scales $\left(k d_{i}<15\right)$, energy is mainly localized on the dispersion relations. The weak nonlinear effects manifest themselves in a broadening of the $\left(\omega-k d_{i}\right)$ distribution with respect to the linear dispersion relations. Not surprisingly, the broadening increases significantly as the angle approaches $90^{\circ}$, for which the linear terms vanish. Interestingly, we also see around $\omega=\omega_{c i}$ significant energy, which reflects the crosscoupling with the ion-cyclotron waves. Beyond $k d_{i} \sim 15$, all the linear high-frequency signal collapses toward low frequency. The fact that this phenomenon occurs at scales similar to those for which we observe the transition from weak to strong wave ion-cyclotron turbulence suggests that the whistlers are locally (in $k$ ) killed by the strongly nonlinear ion-cyclotron fluctuations. To test this idea, we perform an EMHD simulation $(\mathbf{u}=0)$, with everything being equal otherwise (run V). Figure 12(b) is the EMHD version of Fig. 12(a) corresponding to run V. The $x$ component of the generalized Elsässer variables $Z_{+}^{+}$and $Z_{-}^{-}$are, respectively, replaced by the $x$ component of $\mathcal{B}_{+}$ and $\mathcal{B}_{-}$. The acquisition frequency is divided by 10 , with respect to run $\mathrm{V}\left(d t=4 \times 10^{-4} \omega_{c i}\right)$, in order to capture the 


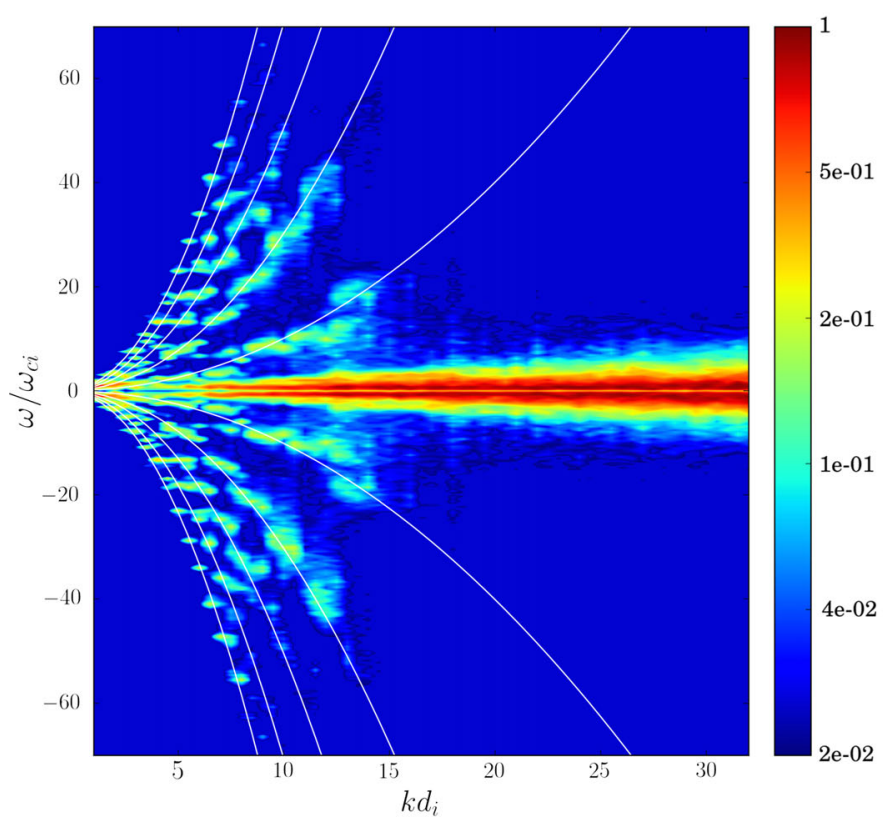

(a)

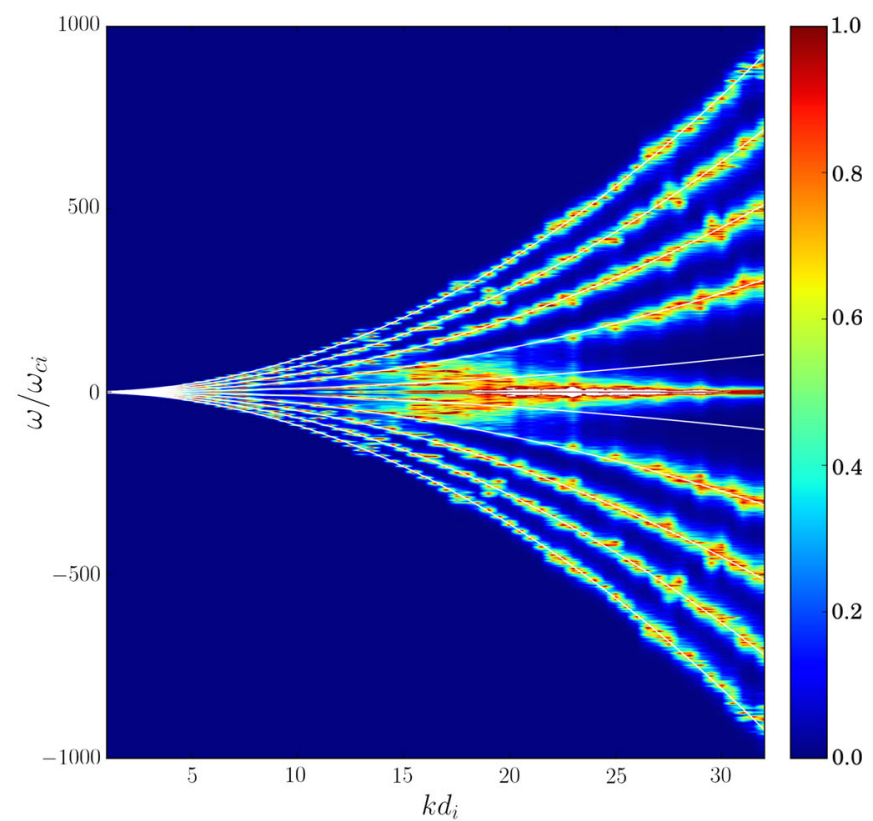

(b)

FIG. 12. (a): Mean of five space-time Fourier spectra of the $x$ component of the generalized Elsässer variables $Z_{+}^{+}$(positive frequency) and $Z_{-}^{-}$(negative frequency) corresponding to five different angles $\theta \sim 84^{\circ}, 72^{\circ}, 60^{\circ}, 45^{\circ}, 25^{\circ}$ (run IV). High frequencies correspond to low angles and reciprocally. Before the mean is taken, each signal is normalized to the maximum value at each fixed $k$. The color scale is $\log _{10} Z_{\Lambda}^{s}(k, \omega)$. Solid white lines are theoretical whistler linear dispersion relations corresponding to the five angles (larger angles correspond to smaller averaged slopes in absolute value). (b) Same as Fig. 12(a) but for the EMHD run V (u $=0$ ). Note that contrary to Fig. 12(a), the color scale is linear; frequency scales are also different.

fastest waves that we observe in this numerical experiment. The result differs strikingly compared to the Hall MHD simulation. We want to draw the reader's attention to the fact that the frequency scales of Figs. 12(a) and 12(b) are different. The whistler fluctuations spread in all of the linearly accessible $k-\omega$ space and demonstrate indirectly that the local (in $k$ ) nonlinear cross-coupling between strongly nonlinear ion-cyclotron fluctuations and whistler waves tends to kill the latter. It is interesting to note, however, that the modes corresponding to an angle of about $84^{\circ}$ do not follow the linear dispersion for all $k$. They experience a transition toward strong turbulence and seem to slightly affect the modes corresponding to an angle of about $72^{\circ}$. They can be seen as a low-frequency, strongly nonlinear, quasi-2D condensate. Remarkably, these strongly interacting wave modes, which are ineluctable as the weak turbulence dynamics develops a strong anisotropy [42], do not significantly affect the dynamics of the weakly interacting one. This situation has also been observed in weak MHD wave turbulence [6,37] and is of primary importance as it validates a posteriori the WTT approach for whistler turbulence.

\section{CONCLUSION}

In this paper, we have considered magnetized plasma turbulence in the framework of incompressible Hall MHD from a WTT perspective [11]. The confrontation of this theory with numerical experiments allowed us to highlight new and interesting properties. We have shown that the ioncyclotron and whistler wave populations are not transparent with respect to each other, as is implicitly assumed in the WTT.

We have shown and explained why Hall MHD weak wave turbulence tends to be a mixture of weakly interacting ioncyclotron and whistler waves embedded in a bath of highly nonlinear ion-cyclotron fluctuations. This situation has a profound impact on the statistical properties. It produces anomalous (with respect to the WTT) scaling and anisotropy.

Using higher-order polyspectra, we have shown that resonant triadic interactions, the building blocks of WTT, survive the bath of highly nonlinear ion-cyclotron fluctuations (Sec. VI). This study allowed us to highlight the importance of resonant interactions involving waves with different polarity, which were neglected in analytical studies of Hall MHD WTT.

The analysis of the space-time Fourier spectra enables us to study the details of nonlinear dynamics (Sec. V). We have seen, in particular, that whistler waves and, consequently, the weak wave turbulent dynamics survive only in the $k$-space region where ion-cyclotron waves are weakly interacting, a situation that limits the domain of applicability of the WTT framework in Hall MHD significantly. 
The main message of the present work is that if different waves coexist in a turbulent environment, they are irredeemably interwoven by the nonlinearities, even if they "live" in remote areas of the frequency domain. As a result, they lose their own identity since they exchange some of their characteristics. We believe that this general observation remains valid and has far-reaching implications for magnetized collisionless plasma turbulence, or turbulence in the presence of multiple interacting waves, in general.

\section{ACKNOWLEDGMENTS}

The research leading to these results has received funding from the European Commission's 7th Framework Program (FP7/2007-2013) under the grant agreement SHOCK (Project No. 284515), from the ANR Contract No. 10JCJC-0403, and ANR-JC project THESOW. The computing resources were made available through the UKMHD Consortium facilities funded by STFC Grant No. ST/ H008810/1. This work was made possible thanks to the HPC resources of TGCC (Très Grand Centre de calcul du CEA) made available by GENCI (Grand Équipement National de Calcul Intensif) (Project No. A0010510117). R. M. acknowledges financial support from the EU-funded Marie-Curie-Skłodowska Global Fellowship (Project No. 661472).

\section{APPENDIX A: HEURISTIC DESCRIPTION OF HALL MHD WTT}

The study of weak wave turbulence in Hall MHD is a difficult task requiring great analytical efforts. Fortunately, it is possible to derive a generalized heuristic description that is able to recover the essential physics underlying the rigorous analytical prediction of WTT [11]. The first step is to introduce the generalized Elsässer variables adapted to Hall MHD. To do so, it turns out that it is necessary to use a complex helical decomposition. Such a decomposition provides a compact description of the dynamics and allows diagonalization of the system dealing with circularly polarized waves. This approach was used to study the dynamics of helicity, inertial, whistler, or magnetostrophic waves [55-60] and is the correct basis for the derivation of the asymptotic equations by Eulerian methods [11].

\section{Helicity basis}

The complex helicity decomposition is defined by

$$
\mathbf{h}^{\Lambda}(\mathbf{k}) \equiv \mathbf{h}_{\mathbf{k}}^{\Lambda}=\hat{\mathbf{e}}_{\theta}+i \Lambda \hat{\mathbf{e}}_{\Phi},
$$

where $i^{2}=-1$,

$$
\hat{\mathbf{e}}_{\theta}=\hat{\mathbf{e}}_{\Phi} \times \hat{\mathbf{e}}_{k}, \quad \hat{\mathbf{e}}_{\Phi}=\frac{\hat{\mathbf{e}}_{\|} \times \hat{\mathbf{e}}_{k}}{\left|\hat{\mathbf{e}}_{\|} \times \hat{\mathbf{e}}_{k}\right|}
$$

and therefore $\left|\hat{\mathbf{e}}_{\theta}\right|=\left|\hat{\mathbf{e}}_{\Phi}\right|=1$. In these relations, the wave vector $\mathbf{k}=k \hat{\mathbf{e}}_{\mathbf{k}}=\mathbf{k}_{\perp}+\mathbf{k}_{\|} \hat{\mathbf{e}}_{\|}$(with $k=|\mathbf{k}|, k_{\perp}=\left|\mathbf{k}_{\perp}\right|$, $\left|\hat{\mathbf{e}}_{\mathbf{k}}\right|=1, \hat{\mathbf{e}}_{\|}$being the direction along $\mathbf{b}_{0}$ ). Here, $\Lambda$ is called the wave polarization, and it takes the values \pm . We note in passing that $\left(\hat{\mathbf{e}}_{\mathbf{k}}, \mathbf{h}_{\mathbf{k}}^{+}, \mathbf{h}_{\mathbf{k}}^{-}\right)$forms a complex basis with the following properties:

$$
\begin{gathered}
\mathbf{h}_{\mathbf{k}}^{-\Lambda}=\mathbf{h}_{-\mathbf{k}}^{\Lambda}, \\
\hat{\mathbf{e}}_{\mathbf{k}} \times \mathbf{h}_{\mathbf{k}}^{\Lambda}=-i \Lambda \mathbf{h}_{\mathbf{k}}^{\Lambda}, \\
\mathbf{k} \cdot \mathbf{h}_{\mathbf{k}}^{\Lambda}=0, \\
\mathbf{h}_{\mathbf{k}}^{\Lambda} \cdot \mathbf{h}_{\mathbf{k}}^{\Lambda^{\prime}}=2 \delta_{-\Lambda^{\prime} \Lambda} .
\end{gathered}
$$

With this decomposition, we see that the incompressibility conditions (2) are automatically satisfied. The Fourier transform of the original vectors $\mathbf{u}(\mathbf{x})$ and $\mathbf{b}(\mathbf{x})$ can be projected on the helicity basis; we write

$$
\begin{aligned}
\hat{\mathbf{u}}(\mathbf{k}) & =\sum_{\Lambda} \mathcal{U}_{\Lambda}(\mathbf{k}) \mathbf{h}_{k}^{\Lambda}, \\
\hat{\mathbf{b}}(\mathbf{k}) & =\sum_{\Lambda} \mathcal{B}_{\Lambda}(\mathbf{k}) \mathbf{h}_{k}^{\Lambda} .
\end{aligned}
$$

\section{Eigenvectors and eigenmodes}

The introduction of Eqs. (A7) and (A8) into the Fourier transform of Eqs. (3) and (4) gives, after the projection on $\mathbf{h}_{-\mathbf{k}}^{\Lambda}$ and linearization,

$$
\begin{gathered}
\partial_{t} \mathcal{U}_{\Lambda}-i b_{0} k_{\|} \mathcal{B}_{\Lambda}=0, \\
\partial_{t} \mathcal{B}_{\Lambda}-i b_{0} k_{\|} \mathcal{U}_{\Lambda}+i \Lambda d_{i} b_{0} k_{\|} k \mathcal{B}_{\Lambda}=0 .
\end{gathered}
$$

To derive the dispersion relation, we introduce the generalized Elsässer fields (the eigenvectors):

$$
\mathcal{Z}_{\Lambda}^{s}=\mathcal{U}_{\Lambda}+\xi_{\Lambda}^{s} \mathcal{B}_{\Lambda}
$$

with $s= \pm$ and

$$
\xi_{\Lambda}^{s}(k)=\xi_{\Lambda}^{s}=-\frac{s d_{i} k}{2}\left(s \Lambda+\sqrt{1+\frac{4}{d_{i}^{2} k^{2}}}\right) .
$$

Then, we obtain

$$
\partial_{t} \mathcal{Z}_{\Lambda}^{s}=-i \omega_{\Lambda}^{s} \mathcal{Z}_{\Lambda}^{s},
$$

with the dispersion relation 


$$
\omega_{\Lambda}^{s}=-b_{0} k_{\|} \xi_{\Lambda}^{s}
$$

Incompressible Hall MHD supports $R$ and $L$ circularly polarized waves, which correspond to (oblique) whistler and ion-cyclotron waves, respectively. We can easily check that in the small-scale limit $\left(k d_{i} \rightarrow+\infty\right)$, we have $\xi_{\Lambda}^{s} \rightarrow$ $-s d_{i} k$ for whistler waves $(\Lambda=s)$ and $\xi_{\Lambda}^{s} \rightarrow-s /\left(d_{i} k\right)$ for ion-cyclotron waves $(\Lambda=-s)$. In the large-scale limit $\left(k d_{i} \rightarrow 0\right)$, we find $\xi_{\Lambda}^{s} \rightarrow-s$, and we recover the classical Elsässer variables used in standard MHD.

\section{Anisotropic Iroshnikov-Kraichnan spectrum of Hall MHD turbulence}

The anisotropic heuristic theory of Hall MHD WTT is given in Ref. [11]. We recall here the main steps of the derivation. The nonlinear time built on the generalized Elsässer variables can be written as

$$
\tau_{\mathrm{nl}} \sim\left(k_{\perp} \mathcal{Z}_{\Lambda}^{s}\right)^{-1} .
$$

The period of Hall MHD waves $\tau_{\omega}$ is given by

$$
\tau_{\omega} \sim\left(\omega_{\Lambda}^{s}\right)^{-1}=-\left(b_{0} k_{\|} \xi_{\Lambda}^{s}\right)^{-1} .
$$

As far as dimensional analysis is concerned, the characteristic transfer time of energy $\tau_{\text {tr }}$ can be an arbitrary function of these eight different characteristic times. Additional physical assumptions are therefore necessary to fix the scaling. In weak wave turbulence, we have the inequality $\tau_{\omega} \ll \tau_{\mathrm{nl}}$, and many stochastic collisions are necessary to significantly modify a wave packet. If we assume that the cumulative perturbation evolves as a random walk, the transfer time becomes $[61,62]$

$$
\tau_{\text {tr }} \sim \tau_{\mathrm{nl}}^{2} / \tau_{w} .
$$

If we now assume a stationary state for which the mean rate of energy dissipation per unit mass $\epsilon$ is independent of the scale, we obtain

$$
\epsilon \sim \frac{E}{\tau_{\text {tr }}} \sim \frac{E\left(k_{\perp}, k_{\|}\right) k_{\perp} k_{\|}}{\tau_{\text {tr }}} \sim \frac{E\left(k_{\perp}, k_{\|}\right) k_{\perp}^{3} \mathcal{Z}_{\Lambda}^{s}}{-B_{0} \xi_{\Lambda}^{s}}
$$

which gives, after some algebra,

$$
E\left(k_{\perp}, k_{\|}\right) \sim \sqrt{\epsilon B_{0}} k_{\perp}^{-2} k_{\|}^{-1 / 2}\left(1+k_{\perp}^{2} d_{i}^{2}\right)^{-1 / 4} .
$$

We recover, in the small-scale limit $\left(k_{\perp} d_{i} \gg 1\right)$, the expected scaling law for whistler as well as ion-cyclotron wave turbulence $E\left(k_{\perp}, k_{\|}\right) \sim k_{\perp}^{-5 / 2} k_{\|}^{-1 / 2}$ and, in the largescale limit $\left(k_{\perp} d_{i} \ll 1\right)$, the Alfvén wave turbulence scaling law in $E\left(k_{\perp}, k_{\|}\right) \sim k_{\perp}^{-2}$. In the latter case, the parallel wave number is a mute variable because of the dynamical decoupling of parallel planes in Fourier space [63]. This prediction is given for the total energy, however, because in the small-scale limit, we have

$$
\xi_{s}^{-s 2} \rightarrow \frac{1}{k^{2} d_{i}^{2}} \quad(L \text { polarity })
$$

and

$$
\xi_{s}^{s} \rightarrow k^{2} d_{i}^{2} \quad(R \text { polarity }) .
$$

We can easily understand from Eq. (A11) that either the magnetic or the velocity field will dominate in Eq. (A19) depending on which waves one considers. Recalling that the magnetic field is tied in the electron flow, whereas the bulk velocity is carried by the heavy ions, one can understand that the emergence of two circularly polarized waves with opposite polarity is fundamentally linked to the microscopic scales with the opposite electric charge of the two species.

\section{APPENDIX B: KINEMATICS}

\section{Wave fluctuations}

In order to define the kinematics for the total energy of the 3D incompressible Hall MHD, we use, for symmetry reasons, the renormalized field $a_{\Lambda}^{s}$ defined by

$$
\mathcal{Z}_{\Lambda}^{s}=\left(\xi_{\Lambda}^{s}-\xi_{\Lambda}^{-s}\right) a_{\Lambda}^{s} e^{-i \omega_{\Lambda}^{s} t} .
$$

We define statistical quantities by introducing the ensemble average denoted $\langle\cdots\rangle$. We define

$$
E^{u}(\mathbf{k}) \equiv \sum_{\Lambda}\left\langle\mathcal{U}_{\Lambda}(\mathbf{k}) \mathcal{U}_{\Lambda}^{*}(\mathbf{k})\right\rangle
$$

and

$$
E^{b}(\mathbf{k}) \equiv \sum_{\Lambda}\left\langle\mathcal{B}_{\Lambda}(\mathbf{k}) \mathcal{B}_{\Lambda}^{*}(\mathbf{k})\right\rangle,
$$

the kinetic and magnetic energy spectra, respectively. The use of expressions (A11) and (B1) leads to

$$
\begin{aligned}
\left\langle\left|\mathcal{U}_{\Lambda}\right|^{2}\right\rangle= & \xi_{\Lambda}^{-2}\left\langle\left|a_{\Lambda}^{+}\right|^{2}\right\rangle+\xi_{\Lambda}^{+2}\left\langle\left|a_{\Lambda}^{-}\right|^{2}\right\rangle-\left\langle a_{\Lambda}^{+} a_{\Lambda}^{-*} e^{i\left(\omega_{\Lambda}^{+}-\omega_{\Lambda}^{-}\right) t}\right\rangle \\
& -\left\langle a_{\Lambda}^{-} a_{\Lambda}^{+*} e^{i\left(\omega_{\Lambda}^{-}-\omega_{\Lambda}^{+}\right) t}\right\rangle
\end{aligned}
$$

and

$$
\begin{aligned}
\left\langle\left|\mathcal{B}_{\Lambda}\right|^{2}\right\rangle= & \left\langle\left|a_{\Lambda}^{+}\right|^{2}\right\rangle+\left\langle\left|a_{\Lambda}^{-}\right|^{2}\right\rangle+\left\langle a_{\Lambda}^{+} a_{\Lambda}^{-*} e^{i\left(\omega_{\Lambda}^{+}-\omega_{\Lambda}^{-}\right) t}\right\rangle \\
& +\left\langle a_{\Lambda}^{-} a_{\Lambda}^{+*} e^{i\left(\omega_{\Lambda}^{-}-\omega_{\Lambda}^{+}\right) t}\right\rangle .
\end{aligned}
$$


Note that the Hermitian symmetry property of a real-valued quantity (conjugate symmetry) is used for the terms in the right-hand side. Then, the total energy spectrum becomes

$$
\begin{aligned}
E^{u}(\mathbf{k})+E^{b}(\mathbf{k})= & \left(\xi_{+}^{-2}+1\right)\left\langle\left|a_{+}^{+}\right|^{2}\right\rangle+\left(\xi_{-}^{+2}+1\right)\left\langle\left|a_{-}^{-}\right|^{2}\right\rangle \\
& +\left(\xi_{+}^{+2}+1\right)\left\langle\left|a_{+}^{-}\right|^{2}\right\rangle+\left(\xi_{-}^{-2}+1\right)\left\langle\left|a_{-}^{+}\right|^{2}\right\rangle .
\end{aligned}
$$

These results lead us to define the left and right polarized kinetic and magnetic energies as

$$
\begin{gathered}
E_{L}^{u}(\mathbf{k}) \equiv \xi_{+}^{+2}\left\langle\left|a_{+}^{-}\right|^{2}\right\rangle+\xi_{-}^{-2}\left\langle\left|a_{-}^{+}\right|^{2}\right\rangle, \\
E_{R}^{u}(\mathbf{k}) \equiv \xi_{+}^{-2}\left\langle\left|a_{+}^{+}\right|^{2}\right\rangle+\xi_{-}^{+2}\left\langle\left|a_{-}^{-}\right|^{2}\right\rangle, \\
E_{L}^{b}(\mathbf{k}) \equiv\left\langle\left|a_{+}^{-}\right|^{2}\right\rangle+\left\langle\left|a_{-}^{+}\right|^{2}\right\rangle, \\
E_{R}^{b}(\mathbf{k}) \equiv\left\langle\left|a_{+}^{+}\right|^{2}\right\rangle+\left\langle\left|a^{-}\right|^{2}\right\rangle .
\end{gathered}
$$

These definitions provide two important relationships at the level of kinematics:

$$
E_{L}^{u}(\mathbf{k})=\xi_{+}^{+2} E_{L}^{b}(\mathbf{k})=\xi_{-}^{-2} E_{L}^{b}(\mathbf{k})
$$

and

$$
E_{R}^{b}(\mathbf{k})=\xi_{+}^{-2} E_{R}^{u}(\mathbf{k})=\xi_{-}^{+2} E_{R}^{u}(\mathbf{k})
$$

Expressions (B11) and (B12) tell us that, in the small-scale limit $k d_{i} \gg 1$, the dynamics is mainly driven by the velocity field for the $L$ fluctuations and by the magnetic field for the $R$ fluctuations. Knowing $E_{L}^{u}(\mathbf{k})$ or $E_{R}^{b}(\mathbf{k})$ automatically gives the form of the spectra for the corresponding fluctuations with the same type of polarity, the latter being driven by the former. Remarkably, this scenario is also applicable in the isotropic case as shown in Ref. [46], for which the concept of polarization was generalized.

\section{Two-dimensional state}

In the different relationships derived above, terms of mixed polarities $\left(\propto e^{i\left(\omega_{\Lambda}^{s}-\omega_{\Lambda}^{-s}\right) t}\right)$ appear. This type of contribution is expected to be weaker than the others (pure real terms) because the presence of a mean magnetic field dynamically leads to the separation between the timescales of amplitudes and phases, thus with a tendency to a statistical cancelation. It is in the weak turbulence limit that the phase mixing is the strongest: In this case, these contributions tend asymptotically to zero. However, the situation is different for the two-dimensional state that corresponds, by definition, to $k_{\|}=0$. For this state, we may simplify the terms of the mixed polarities because we have $\omega_{\Lambda}^{s}=0$. The phase mixing does not operate at all, and a significant contribution to the kinematics of the mixed polarity terms may be expected.

[1] D. J. Benney and A. C. Newell, Statistical Properties of the Sea, Phys. Fluids 10, S281 (1967).

[2] K. Hasselmann, On the Non-linear Energy Transfer in Gravity-Wave Spectrum: Part 1. General Theory, J. Fluid Mech. 12, 481 (1962).

[3] A. A. Vedenov, Theory of Weakly Turbulent Plasma, Reviews of Plasma Physics, edited by M. A. Leontovich (Springer, Boston, MA, 1967).

[4] S. Nazarenko and M. Onorato, Wave Turbulence and Vortices in Bose-Einstein Condensation, Physica D (Amsterdam) 219, 1 (2006).

[5] S. Galtier and S. V. Nazarenko, Turbulence of Weak Gravitational Waves in the Early Universe, Phys. Rev. Lett. 119, 221101 (2017).

[6] R. Meyrand, K. H. Kiyani, and S. Galtier, Weak Magnetohydrodynamic Turbulence and Intermittency, J. Fluid Mech. 770, R1 (2015).

[7] Y. Lvov, S. Nazarenko, and R. West, Wave Turbulence in Bose-Einstein Condensates, Physica D (Amsterdam) 184, 333 (2003).

[8] S. Dyachenko, A.C. Newell, A. Pushkarev, and V.E. Zakharov, Optical Turbulence: Weak Turbulence, Condensates and Collapsing Filaments in the Nonlinear Schrödinger Equation, Physica D (Amsterdam) 57, 96 (1992).

[9] Q. Aubourg and N. Mordant, Nonlocal Resonances in Weak Turbulence of Gravity-Capillary Waves, Phys. Rev. Lett. 114, 144501 (2015).

[10] N. Mordant, Are There Waves in Elastic Wave Turbulence?, Phys. Rev. Lett. 100, 234505 (2008).

[11] S. Galtier, Wave Turbulence in Incompressible Hall Magnetohydrodynamics, J. Plasma Phys. 72, 721 (2006).

[12] A. Ito, A. Hirose, S. M. Mahajan, and S. Ohsaki, Remarks on the Discrete Alfven Wave Spectrum Induced by the Hall Current, Phys. Plasmas 11, 5643 (2004).

[13] A. Hirose, A. Ito, S. M. Mahajan, and S. Ohsaki, Relation between Hall-Magnetohydrodynamics and the Kinetic Alfuén Wave, Phys. Lett. A 330, 474 (2004).

[14] M. W. Kunz and G. Lesur, Magnetic Self-Organisation in Hall-Dominated Magnetorotational Turbulence, Mon. Not. R. Astron. Soc. 434, 2295 (2013).

[15] K. N. Gourgouliatos and A. Cumming, Hall Attractor in Axially Symmetric Magnetic Fields in Neutron Star Crusts, Phys. Rev. Lett. 112, 171101 (2014).

[16] C. M. Cooper, J. Wallace, M. Brookhart, M. Clark, C. Collins, W. X. Ding, K. Flanagan, I. Khalzov, Y. Li, J. Milhone, M. Nornberg, P. Nonn, D. Weisberg, D. G. Whyte, E. Zweibel, and C. B. Forest, The Madison Plasma Dynamo Experiment: A Facility for Studying Laboratory Plasma Astrophysics, Phys. Plasmas 21, 013505 (2014).

[17] C. B. Forest, K. Flanagan, M. Brookhart, M. Clark, C. M. Cooper, V. Désangles, J. Egedal, D. Endrizzi, I. V. Khalzov, H. Li, M. Miesch, J. Milhone, M. Nornberg, J. Olson, E. Peterson, F. Roesler, A. Schekochihin, O. Schmitz, R. Siller, 
A. Spitkovsky et al., The Wisconsin Plasma Astrophysics Laboratory, J. Plasma Phys. 81, 345810501 (2015).

[18] Y. Ren, M. Yamada, S. Gerhardt, H. Ji, R. Kulsrud, and A. Kuritsyn, Experimental Verification of the Hall Effect During Magnetic Reconnection in a Laboratory Plasma, Phys. Rev. Lett. 95, 055003 (2005).

[19] Y. Ren, M. Yamada, H. Ji, S. Dorfman, S. P. Gerhardt, and R. Kulsrud, Experimental Study of the Hall Effect and Electron Diffusion Region During Magnetic Reconnection in a Laboratory Plasma, Phys. Plasmas 15, 082113 (2008).

[20] D. T. Garnier, A. Hansen, M. E. Mauel, E. Ortiz, A. C. Boxer, J. Ellsworth, I. Karim, J. Kesner, S. Mahar, and A. Roach, Production and Study of High-Beta Plasma Confined by a Superconducting Dipole Magnet, Phys. Plasmas 13, 056111 (2006).

[21] J. y. Shiraishi, M. Furukawa, and Z. Yoshida, A New-Type Equilibrium of Flowing Plasma Dominated by the Hall Effect, Plasma Fusion Research 1, 050 (2006).

[22] G. G. Howes, Limitations of Hall MHD as a Model for Turbulence in Weakly Collisional Plasmas, Nonlinear Processes Geophys. 16, 219 (2009).

[23] A. A. Schekochihin, S. C. Cowley, W. Dorland, G. W. Hammett, G. G. Howes, E. Quataert, and T. Tatsuno, Astrophysical Gyrokinetics: Kinetic and Fluid Turbulent Cascades in Magnetized Weakly Collisional Plasmas, Astrophys. J. Suppl. Ser. 182, 310 (2009).

[24] E. Marsch and C. Y. Tu, Spectral and Spatial Evolution of Compressible Turbulence in the Inner Solar Wind, J. Geophys. Res. 95, 11945 (1990).

[25] P. J. Kellogg and T. S. Horbury, Rapid Density Fluctuations in the Solar Wind, Ann. Geophys. 23, 3765 (2005).

[26] F. Sahraoui, M. L. Goldstein, P. Robert, and Yu. V. Khotyaintsev, Evidence of a Cascade and Dissipation of Solar-Wind Turbulence at the Electron Gyroscale, Phys. Rev. Lett. 102, 231102 (2009).

[27] O. Alexandrova, J. Saur, C. Lacombe, A. Mangeney, J. Mitchell, S. J. Schwartz, and P. Robert, Universality of Solar-Wind Turbulent Spectrum from MHD to Electron Scales, Phys. Rev. Lett. 103, 165003 (2009).

[28] O. Chang, S. Peter Gary, and J. Wang, Whistler Turbulence Forward Cascade: Three-Dimensional Particle-in-Cell Simulations, Geophys. Res. Lett. 38, 22102 (2011).

[29] J. Maron and P. Goldreich, Simulations of Incompressible Magnetohydrodynamic Turbulence, Astrophys. J. 554, 1175 (2001).

[30] C. H. K. Chen, L. Leung, S. Boldyrev, B. A. Maruca, and S. D. Bale, Ion-Scale Spectral Break of Solar Wind Turbulence at High and Low Beta, Geophys. Res. Lett. 41, 8081 (2014).

[31] P. Hunana, D. Laveder, T. Passot, P. L. Sulem, and D. Borgogno, Reduction of Compressibility and Parallel Transfer by Landau Damping in Turbulent Magnetized Plasmas, Astrophys. J. 743, 128 (2011).

[32] S. Galtier and R. Meyrand, Entanglement of Helicity and Energy in Kinetic Alfvén Wave/Whistler Turbulence, J. Plasma Phys. 81, 325810106 (2015).

[33] B. Teaca, M. K. Verma, B. Knaepen, and D. Carati, Energy Transfer in Anisotropic Magnetohydrodynamic Turbulence, Phys. Rev. E 79, 046312 (2009).
[34] S. M. Mahajan and V. Krishan, Exact Solution of the Incompressible Hall Magnetohydrodynamics, Mon. Not. R. Astron. Soc. 359, L27 (2005).

[35] R. Meyrand, Ph.D. thesis, Paris-Sud University (2013).

[36] M. K. Williamson, Low-Storage Runge-Kutta Schemes, J. Comput. Phys. 35, 48 (1980).

[37] R. Meyrand, S. Galtier, and K. H. Kiyani, Direct Evidence of the Transition from Weak to Strong Magnetohydrodynamic Turbulence, Phys. Rev. Lett. 116, 105002 (2016).

[38] S. Nazarenko, 2D Enslaving of MHD Turbulence, New J. Phys. 9, 307 (2007).

[39] J. Cho and A. Lazarian, The Anisotropy of Electron Magnetohydrodynamic Turbulence, Astrophys. J. 615, L41 (2004).

[40] C. S. Ng, A. Bhattacharjee, K. Germaschewski, and S. Galtier, The Anisotropy of Electron Magnetohydrodynamic Turbulence, Phys. Plasmas 10, 1954 (2003).

[41] J. Cho and A. Lazarian, Simulations of Electron Magnetohydrodynamic Turbulence, Astrophys. J. 701, 236 (2009).

[42] R. Meyrand and S. Galtier, Anomalous $k_{\perp}^{-8 / 3}$ Spectrum in Electron Magnetohydrodynamic Turbulence, Phys. Rev. Lett. 111, 264501 (2013).

[43] F. Sahraoui, G. Belmont, and M. L. Goldstein, New Insight into Short-Wavelength Solar Wind Fluctuations from Vlasov Theory, Astrophys. J. 748, 100 (2012).

[44] S. Dastgeer, A. Das, P. Kaw, and P. H. Diamond, Hydrodynamic Regime of Two-Dimensional Electron Magnetohydrodynamics, Phys. Plasmas 7, 571 (2000).

[45] S. Galtier and A. Bhattacharjee, Anisotropic Weak Whistler Wave Turbulence in Electron Magnetohydrodynamics, Phys. Plasmas 10, 3065 (2003).

[46] R. Meyrand and S. Galtier, Spontaneous Chiral Symmetry Breaking of Hall MHD Turbulence, Phys. Rev. Lett. 109, 194501 (2012).

[47] P. D. Mininni, A. Alexakis, and A. Pouquet, Energy Transfer in Hall-MHD Turbulence: Cascades, Backscatter, and Dynamo Action, J. Plasma Phys. 73, 377 (2007).

[48] S. Galtier, Wave Turbulence in Magnetized Plasmas, Nonlinear Processes Geophys. 16, 83 (2009).

[49] S. D. Bale, D. Burgess, P. J. Kellogg, K. Goetz, R. L. Howard, and S. J. Monson, Evidence of Three-Wave Interactions in the Upstream Solar Wind, Geophys. Res. Lett. 23, 109 (1996).

[50] T. D. de Wit and V. V. Krasnoselskikh, Wavelet Bicoherence Analysis of Strong Plasma Turbulence at the Earth's Quasiparallel Bow Shock, Phys. Plasmas 2, 4307 (1995).

[51] Ch. P. Ritz, E. J. Powers, T. L. Rhodes, R. D. Bengtson, K. W. Gentle, H. Lin, P. E. Phillips, A. J. Wootton, D. L. Brower, N. C. Luhmann, W. A. Peebles, P. M. Schoch, and R. L. Hickok, Advanced Plasma Fluctuation Analysis Techniques and Their Impact on Fusion Research, Rev. Sci. Instrum. 59, 1739 (1988).

[52] P. H. Diamond, M. N. Rosenbluth, E. Sanchez, C. Hidalgo, B. van Milligen, T. Estrada, B. Brañas, M. Hirsch, H. J. Hartfuss, and B. A. Carreras, In Search of the Elusive Zonal Flow Using Cross-Bicoherence Analysis, Phys. Rev. Lett. 84, 4842 (2000).

[53] C. H. Mccomas and M. G. Briscoe, Bispectra of Internal Waves, J. Fluid Mech. 97, 205 (1980). 
[54] L. Verde, A. F. Heavens, W. J. Percival, S. Matarrese, C. M. Baugh, J. Bland-Hawthorn, T. Bridges, R. Cannon, S. Cole, M. Colless, C. Collins, W. Couch, G. Dalton, R. De Propris, S. P. Driver, G. Efstathiou, R. S. Ellis, C. S. Frenk, K. Glazebrook, C. Jackson et al., The 2dF Galaxy Redshift Survey: The Bias of Galaxies and the Density of the Universe, Mon. Not. R. Astron. Soc. 335, 432 (2002).

[55] A. Craya, Contribution à l'analyse de la Turbulence Associée à des Vitesse Moyennes, https://tel.archives-ouvertes.fr/tel00684659/file/Craya300-OCR.pdf.

[56] H. K. Moffatt, Dynamo Action Associated with Random Inertial Waves in a Rotating Conducting Fluid, J. Fluid Mech. 44, 705 (1970).

[57] R. H. Kraichnan, Helical Turbulence and Absolute Equilibrium, J. Fluid Mech. 59, 745 (1973).

[58] F. Waleffe, The Nature of Triad Interactions in Homogeneous Turbulence, Phys. Fluids 4, 350 (1992).
[59] S. Galtier, Weak Inertial-Wave Turbulence Theory, Phys. Rev. E 68, 015301 (2003).

[60] S. Galtier, Weak Turbulence Theory for Rotating Magnetohydrodynamics and Planetary Flows, J. Fluid Mech. 757, 114 (2014).

[61] P. S. Iroshnikov, Turbulence of a Conducting Fluid in a Strong Magnetic Field, Sov. Astron. 7, 566 (1964).

[62] R. H. Kraichnan, Inertial Range Spectrum in Hydromagnetic Turbulence, Phys. Fluids 8, 1385 (1965).

[63] S. Galtier, S. V. Nazarenko, A. C. Newell, and A. Pouquet, A Weak Turbulence Theory for Incompressible Magnetohydrodynamics, J. Plasma Phys. 63, 447 (2000).

Correction: Article components on page 14 of the PDF version were overlaid, making portions unreadable, and have been fixed. 